Draft VERsion MAY 28, 2022

Preprint typeset using $\mathrm{IAT}_{\mathrm{E}} \mathrm{X}$ style emulateapj v. 12/16/11

\title{
THE MAGNETIC SENSITIVITY OF THE RESONANCE AND SUBORDINATE LINES OF MG II IN THE SOLAR CHROMOSPHERE
}

\author{
T. Del Pino Alemán ${ }^{1,2}$, J. Trujillo Bueno ${ }^{1,2,3, a}$, R. Casini ${ }^{4}$, And R. Manso Sainz ${ }^{5}$ \\ ${ }^{1}$ Instituto de Astrofísica de Canarias, E-38205 La Laguna, Tenerife, Spain \\ ${ }^{2}$ Departamento de Astrofísica, Universidad de La Laguna, E-38206 La Laguna, Tenerife, Spain \\ ${ }^{3}$ Consejo Superior de Investigaciones Científicas, Spain \\ ${ }^{4}$ High Altitude Observatory, National Center for Atmospheric Research ${ }^{b}$. \\ P.O. Box 3000, Boulder, CO 80307-3000, U.S.A. \\ and \\ ${ }^{5}$ Max-Planck-Institut für Sonnensystemforschung, \\ Justus-von-Liebig-Weg 3, 37077 Göttingen, Germany \\ Draft version May 28, 2022
}

\begin{abstract}
We carry out a theoretical study of the polarization of the solar Mg II h-k doublet (including its extended wings) and the subordinate UV triplet around $280 \mathrm{~nm}$. These lines are of great diagnostic interest, as they encode information on the physical properties of the solar atmosphere from the upper photosphere to the chromosphere-corona transition region. We base our study on radiative transfer calculations of spectral line polarization in one-dimensional models of quiet and plage regions of the solar atmosphere. Our calculations take into account the combined action of atomic polarization, quantum level interference, frequency redistribution, and magnetic fields of arbitrary strength. In particular, we study the sensitivity of the emergent Stokes profiles to changes in the magnetic field through the Zeeman and Hanle effects. We also study the impact of the chromospheric plasma dynamics on the emergent Stokes profiles, taking into account the angle-dependent frequency redistribution in the h-k resonance transitions. The results presented here are of interest for the interpretation of spectropolarimetric observations in this important region of the solar ultraviolet spectrum.
\end{abstract}

Subject headings: line: profiles - polarization - scattering - radiative transfer - Sun: chromosphere

\section{INTRODUCTION}

The magnetic field plays a key role in determining the behavior of the solar plasma. In the outer layers of the solar atmosphere, i.e., the chromosphere, the transition region, and the corona, the fast decrease in density is such that the magnetic field dominates the structuring of the low- $\beta$ plasma (e.g., Priest 2014). The determination of the magnetic fields in these regions of the solar atmosphere is one of the main challenges faced in solar physics nowadays.

The polarization profiles of spectral lines carry information on many physical properties of the emitting plasma, in particular, of the magnetic field strength and geometry. Therefore, by studying the polarization of the electromagnetic radiation emerging from the solar atmosphere we can aspire to determine its magnetic properties.

Most of the atomic lines in the visible solar spectrum are formed in the lower layers of the solar atmosphere. In order to study the magnetic fields in the outer atmospheric regions, we have to look for spectral lines that are formed there, many of which are strong resonance lines in the ultraviolet (UV) region of the solar spectrum. The challenge of diagnosing the magnetic field is then twofold.

First, UV observations are challenging or simply impossible from ground-based observing facilities due to the absorption of the Earth atmosphere. Therefore, space telescopes are preferable or necessary to observe many UV atomic lines of diagnostic relevance. In 2013, NASA launched the Interface Region Imaging Spectrograph (IRIS; see De Pontieu et al. 2014), which continues to provide excellent spectroscopic observations of UV lines such as Mg II h-k (e.g., Pereira et al. 2014). Two years later, the Chromospheric Lyman Alpha Spectro-Polarimeter sounding rocket experiment (CLASP; Kano et al. 2012, Kobayashi et al. 2012) provided the first on-disk observations of the linear polarization spectrum of the hydrogen Lyman- $\alpha$ line (Kano et al. 2017). Theoretical modeling of those observations has allowed to constrain the geometric complexity of the chromosphere-corona transition region (Trujillo Bueno et al. 2018). Very recently, while this paper was being written, NASA launched with success the Chromospheric LAyer Spectro-Polarimeter (CLASP-2; Narukage et al. 2016) to measure the intensity and polarization across the Mg II h-k lines with high polarimetric sensitivity and spectral resolution, in both quiet and active regions of the solar disk. More than three decades ago, the Ultra-Violet Spectro-Polarimeter (UVSP; Calvert et al. 1979;: Woodgate et al. 1980) on board the Solar Maximum Mission (SMM; Bohlin et al. 1980; Strong et al. 1999) satellite was able to detect $Q / I$ scattering signals of the order of a few percent in the far wings of the Mg II h-k lines (Henze \& Stenflo 1987). Such large $Q / I$ wing signals, as well as the negative (radial) scattering polarization in the region between the $\mathrm{h}$ and $\mathrm{k}$ lines, had been theoretically predicted by Auer et al. (1980) using the approximation of coherent scattering in the observer's frame. Stenflo (1980) observed such Q/I pattern across the Ca II H and K resonance lines and pointed out that it is

a Affiliate scientist of the National Center for Atmospheric Research, Boulder, U.S.A.

b The National Center for Atmospheric Research is sponsored by the National Science Foundation. 
due to quantum mechanical interference between the sublevels pertaining to the upper $J$ levels of the $\mathrm{H}$ and $\mathrm{K}$ lines. Evidence for the expected negative polarization at wing wavelengths between the $\mathrm{Mg}$ II resonance lines has indeed been found in a reanalysis of the observations from SMM (Manso Sainz et al. 2019).

Second, because of the rapid decrease of the density with height in the solar atmosphere, collisional processes become less important in the statistical equilibrium of the ion compared to radiative processes. Moreover, as photons can more easily escape from the plasma, the radiation field becomes more and more anisotropic. This anisotropic radiation field produces atomic polarization, that is, population imbalances and coherence among the magnetic sublevels of the atom. The differential absorption and emission of radiation in the presence of atomic polarization is able to produce linear polarization - the so-called line scattering polarization - even in the absence of magnetic fields. A magnetic field splits the energy of the magnetic sublevels (Zeeman effect) and modifies the scattering polarization by relaxing the atomic level coherence (Hanle effect). The lower rate of collisional processes also implies that the frequency correlation between absorbed and re-emitted photons in the scattering events becomes relevant in the line formation process, giving rise to the so-called partial frequency redistribution (PRD) regime. Both lines observed by the CLASP experiments are affected by PRD effects and by quantum mechanical interference between the two upper levels of those transitions (Belluzzi \& Trujillo Bueno 2012, Belluzzi et al. 2012, Kano et al. 2017).

Clearly, the interpretation of spectropolarimetric observations of strong UV resonance lines requires a thorough theoretical understanding - and a comparatively adequate numerical modeling - of the generation and transfer of spectral line polarized radiation in magnetized plasmas, (see the review by Trujillo Bueno et al. 2017). For instance, the Lyman- $\alpha$ and Mg II h-k lines result from transitions between the ground term, composed of the single level ${ }^{2} \mathrm{~S}_{1 / 2}$ and the first excited term, composed of the ${ }^{2} \mathrm{P}_{1 / 2}$ and ${ }^{2} \mathrm{P}_{3 / 2}$ levels. A rigorous radiative transfer modeling requires taking into account correlation effects between the incoming and outgoing photons in the scattering events (PRD effects), as well as the effects of quantum interference between the two upper levels with $J=3 / 2$ and $J=1 / 2$. This radiative transfer problem has been solved by Belluzzi \& Trujillo Bueno (2012, 2014) for the Mg II h-k lines and by Belluzzi et al. (2012) for the Lyman- $\alpha$ line using one-dimensional (1D) model atmospheres without magnetic fields. These authors demonstrated that PRD effects and $J$-state interference have a very important impact outside the center of the lines, and that the approximation of coherent scattering in the observer's frame used by Auer et al. (1980) to investigate the scattering polarization in the wings of $\mathrm{Mg}$ II h-k produces a significant overestimation of the polarization amplitudes. They also showed that the approximation of complete frequency redistribution (CRD) without $J$-state interference used by Trujillo Bueno et al. (2011) is suitable for estimating the line-center scattering polarization where the Hanle effect operates. 'The impact of the joint action of the Hanle, Zeeman, and PRD effects has been investigated by Alsina Ballester et al. (2016) for the Mg II k line (using a two-level atomic model, therefore without $J$-state interference) and by del Pino Alemán et al. (2016) for the Mg II h-k lines (using a two-term atomic model, therefore with $J$-state interference). These papers highlighted that the $\rho_{V} Q$ and $\rho_{V} U$ magneto-optical (M-O) terms of the transfer equations for Stokes $U$ and $Q$, respectively, give rise to very significant signals in the wings of $U / I$. The two-term atom investigation by del Pino Alemán et al. (2016) showed that the linear polarization is sensitive to the magnetic field all over the wings of the $\mathrm{Mg}$ II resonance lines.

These studies on the polarization of the Mg II resonance lines accounting for PRD effects and scattering polarization were carried out using atomic models that only included either or both the $\mathrm{h}$ and $\mathrm{k}$ lines. However, the same spectral region also contains a triplet of subordinate lines of $\mathrm{Mg}$ II (see Fig. 1), which also are of diagnostic interest (see Belluzzi \& Trujillo Bueno 2012; Pereira et al. 2015). One of the lines in such triplet is located in the blue wing of the $\mathrm{k}$ line, while the other two transitions are blended and located in the red wing of the $\mathrm{k}$ line. Interestingly, the heights of line-center optical depth unity in such subordinate lines are located about $600 \mathrm{~km}$ below those corresponding to the $\mathrm{h}$ and $\mathrm{k}$ line centers (see Fig. 1 of Belluzzi \& Trujillo Bueno 2012). As seen in Fig. 1 the lower levels of this triplet around 279.16 and $279.88 \mathrm{~nm}$ are the upper levels of the h-k doublet. Because this is a cascade transition, rather than a three-term transition of the $\Lambda$-type, the theoretical approach outlined in Casini \& Manso Sainz (2016a) (see also Casini et al. 2017a b) can only be applied neglecting PRD effects in the subordinate lines. Nevertheless, the CRD approximation is valid for modeling the intensity profiles of this triplet (Pereira et al. 2015), so we are confident that this approximation is also suitable for modeling their polarization given that such lines are weaker than the doublet and form lower in the atmosphere.

After presenting the formulation of the problem in 92 , including information about the numerical approach and the atomic and atmospheric models adopted, in \$3 we focus on a detailed presentation of the results. In particular, by using semi-empirical models of quiet and plage regions of the solar disk, we study in depth the thermal and magnetic sensitivity of the polarization of the emergent spectral line radiation. Since the solar chromosphere is highly dynamic, we also pay particular attention to the sensitivity of the intensity and polarization across the Mg II lines to the dynamical state of the plasma. To this end, we have carried out additional radiative transfer calculations in a 1D model of chromospheric dynamics developed by Carlsson \& Stein (1997). In particular, we solved the non-equilibrium polarization transfer problem at each time step of the hydrodynamic simulation, taking into account the effects of angle-dependent frequency redistribution. Finally, in $\$ 4$ we present our main conclusions, including an outlook of future research.

\section{FORMULATION OF THE PROBLEM}

We solve the radiation transfer problem with polarization for the three-term model atom (Fig. 1) underlying the formation of the Mg II h-k doublet at 279.64 and $280.35 \mathrm{~nm}$ and the UV triplet at 279.16 and $279.88 \mathrm{~nm}$. Our objective 


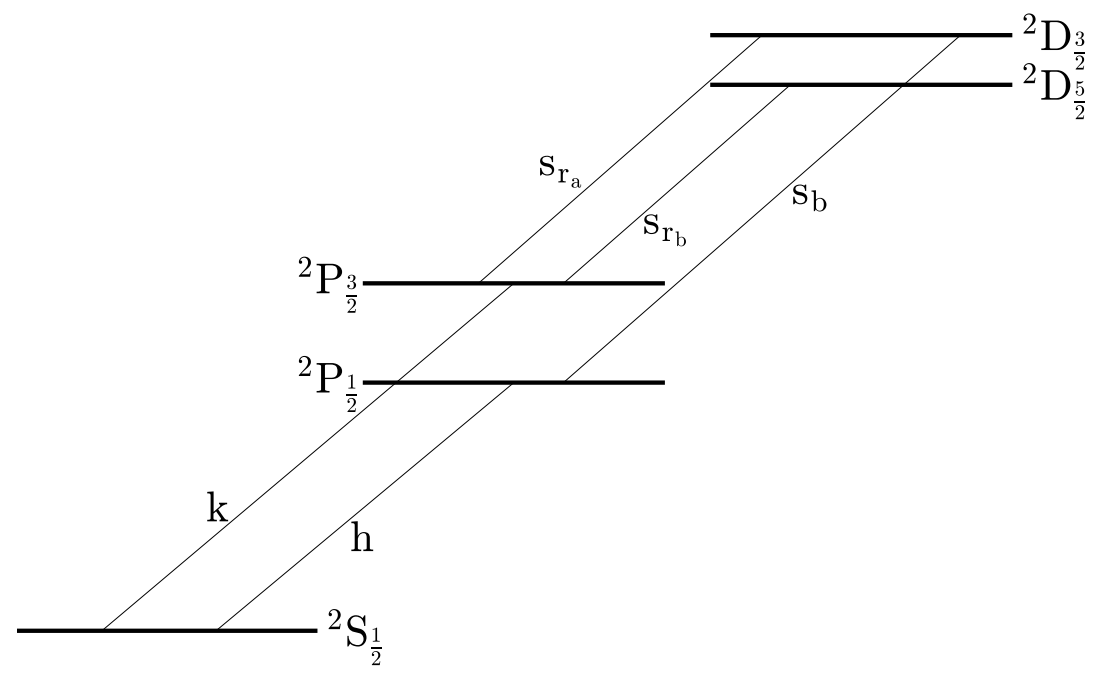

FIG. 1. - Energy diagram of the three-term (S,P,D) atomic model of Mg II adopted for this work. The ground state of Mg III is also taken into account for solving the radiative and collisional statistical equilibrium of the system of transitions shown in the figure. The corresponding four visible spectral lines are: $\mathrm{k}(279.64 \mathrm{~nm}), \mathrm{h}(280.35 \mathrm{~nm}), \mathrm{s}_{\mathrm{b}}(279.16 \mathrm{~nm})$, and $\mathrm{s}_{\mathrm{a}}+\mathrm{s}_{\mathrm{r}}(279.88 \mathrm{~nm})$. Our numerical model fully takes into account quantum interference in the $\mathrm{P}$ and $\mathrm{D}$ terms due to their fine structure, as well as magnetic-induced, level-crossing interference.

is to study the sensitivity of these lines to the magnetic field and to the plasma velocity gradients. We consider several 1D atmospheric models (semi-empirical models and a hydrodynamical time-dependent model) without assuming local thermodynamical equilibrium (hereafter, NLTE), and taking into account scattering polarization (both in the lines and in the continuum), partial frequency redistribution effects, and the Zeeman and Hanle effects.

\subsection{Solution Method}

In order to solve the NLTE problem of the generation and transfer of polarized radiation in an optically thick and magnetized plasma, we must solve, simultaneously, the radiation transfer (RT) equations and the statistical equilibrium (SE) equations. The former determine how the polarized radiation is absorbed and emitted at every point in the plasma, while the latter determine the excitation state of the atom, that is, the populations of the atomic levels and the quantum coherence among them.

We follow the same approach as in del Pino Alemán et al. (2016). Therefore, we consider the SE equations to perturbative first order in the atom-photon interaction (see Landi Degl'Innocenti \& Landolfi 2004), and we solve the RT equations taking into account PRD effects in the transitions $h$ and $k$. Because the lower term of the transitions $\mathrm{S}_{\mathrm{b}}, \mathrm{S}_{\mathrm{r}_{\mathrm{a}}}$, and $\mathrm{s}_{\mathrm{r}_{\mathrm{b}}}$ is also the upper term of $\mathrm{h}$ and $\mathrm{k}$, our modeling framework cannot account for coherent scattering effects in their formation (Casini \& Manso Sainz 2016b). However, because PRD effects are typically only important for resonance transitions (in the case of $\mathrm{Mg}$ II, the $\mathrm{h}$ and $\mathrm{k}$ lines), it is resonable to assume that complete complete redistribution $(\mathrm{CRD})$ is a suitable approximation for the subordinate transitions. Regardless, this approximation needs to be justified once a self-consistent theory becames available to handle this type of atomic system.

Concerning the iterative method of solution, we first solve the unpolarized radiative transfer problem applying the accelerated Lambda iteration method (e.g., Rybicki \& Hummer 1991 and references therein). Once the self-consistent solution is obtained for the populations and intensity, we then apply $\Lambda$-iteration (e.g., Mihalas 1970) for obtaining the density matrix elements and the polarization profiles, initializing the problem with the previously obtained unpolarized solution. This strategy allows to obtain the self-consistent solution for the Stokes profiles after only a few $\Lambda$-iterations (Trujillo Bueno \& Manso Sainz 1999). In a standard quad-core laptop using 5 processes a typical solution of the non-magnetic problem with $\sim 10^{3}$ frequency nodes and 8 quadrature directions takes of the order of 2.5 minutes, while a typical magnetic solution with $\sim 10^{3}$ frequency nodes and 64 quadrature directions takes of the order of $10^{2}$ minutes, both of them with the angle-averaged approximation. With 32 processes in a cluster, a typical solution of the non-magnetic dynamic case with angle-dependent redistribution took of the order of 1 hour, although some snapshots could take up to 3 hours depending on the particular stratification ${ }^{3}$

\footnotetext{
${ }^{3}$ Because the velocity in the time series we use is vertical, we can take advantage of the axial symmetry of the problem, which decreases significantly the computational cost. If this was not the case, the computing time would be at least two orders of magnitude larger.
} 


\subsection{The Atomic and Atmospheric Models}

We solve numerically the NLTE problem of the generation and transfer of polarized radiation in several 1D planeparallel models of the solar atmosphere. We chose the static models C and P of Fontenla et al. (1993, hereafter FAL-C and FAL-P models, respectively), representative of the average quiet Sun and a plage region, respectively. We also calculate the polarized spectrum in the strongly dynamic time-dependent hydrodynamical model of Carlsson \& Stein (1997; hereafter CS model). Fig. 2 shows the variation with height of the temperature, neutral hydrogen number density, vertical velocity, and microturbulent velocity in the mentioned atmospheric models. In the radiative transfer calculations in the static FAL-C and FAL-P models we have taken into account the spectral line broadening produced by the model's microturbulent velocity. No microturbulent velocity is specified for the dynamic CS model, but in our calculations we have chosen a value of $v_{\text {micro }}=7 \mathrm{~km} / \mathrm{s}$, intermediate between the microturbulence at the two formation heights of the doublet and triplet in the FAL-C model.

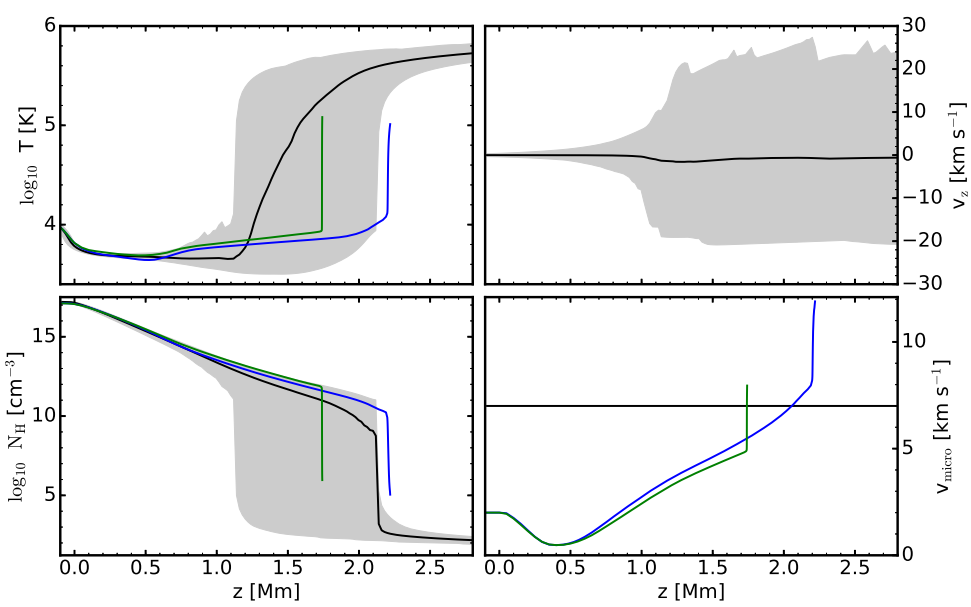

FIG. 2.- Variation with height of the temperature (top left), vertical velocity (top right), H I number density (bottom left), and microtubulent velocity (bottom right), for the model atmospheres used in this work. The black curve is the time-averaged value of the CS model, and the shaded areas represent the span of all the possible variations for the full time series over the full range of heights. The colored curves are for the static models FAL-C (blue) and FAL-P (green).

We used a model atom that includes the three terms of $\mathrm{Mg}$ II relevant for the transitions in the spectral range of interest (see Fig. 1) and the ground term of Mg III. We verified that the emergent Stokes profiles are practically indistinguishable whether we include just these four terms or use a more complex atomic model with eight terms, considering CRD for all the transitions but the h-k doublet. Consequently, we have adopted the smallest atomic model to reduce the computational cost of this investigation.

The energy values of the atomic levels are taken from the NIST database (Kramida et al. 2018). The Einstein $A$-coefficient for spontaneous emission of the transition between two terms of orbital angular momenta $L_{u}$ and $L_{\ell}$ is the weighted average of the corresponding coefficients of the transitions between the atomic $J$-levels within the terms, which are also taken from the NIST database:

$$
A_{L_{u} L_{\ell}}=\frac{1}{\left(2 L_{u}+1\right)(2 S+1)} \sum_{J_{u} J_{\ell}}\left(2 J_{u}+1\right) A_{J_{u} J_{\ell}},
$$

where $J_{\ell}$ and $J_{u}$ are the total angular momenta of the lower and upper levels, respectively, and $S$ is the spin angular momentum. The photoionization cross-sections for the ${ }^{2} \mathrm{~S}$ and ${ }^{2} \mathrm{P}$ terms are taken from the TOPbase database (Cunto et al. 1993) and the one corresponding to the ${ }^{2} \mathrm{D}$ term is taken as hydrogenic (e.g., Mihalas 1978). The collisional excitation rates are taken from Sigut \& Pradhan (1995). Inelastic and super-elastic collisions in the multi-term atom are implemented similarly to the formalism of Belluzzi et al. (2013). Because Sigut \& Pradhan (1995) provide the collisional rates between fine structure levels, we compute the collisional rate between terms by applying Eq. (1) with the substitution $A \rightarrow C$. The bound-free inelastic collisional rates with electrons are computed using the approximation given in Allen (1963). The rates of depolarizing collisions with neutral hydrogen are taken from Manso Sainz et al. (2014).

\section{RESULTS}

Due to the computational cost of calculating the redistribution function for every pair of virtually absorbed-emitted photon frequencies and propagation directions, the synthesis problem with PRD effects is usually solved under the angle-average approximation (e.g., Mihalas 1970 Belluzzi \& Trujillo Bueno 2012). The angle-average approximation is a good one for unmagnetized models, but this is not generally the case for magnetized model atmospheres (see Sampoorna et al. 2017 for a recent study based on academic lines in isothermal model atmospheres). For simplicity and computational time reasons we have used the angle-average approximation for our investigation with magnetic fields in 
the FAL-C and FAL-P models. This is justified because accurate angle-dependent PRD numerical calculations in the presence of inclined fields are very costly, and the aim of this paper is not to model spectropolarimetric observations. However, we relaxed it to derive the results of $\$ 3.3$, where we consider a time-dependent unmagnetized model of solar chromospheric dynamics.

\subsection{Thermal sensitivity}

As shown by Belluzzi \& Trujillo Bueno (2012), PRD effects and quantum interference in the upper term of the $\mathrm{Mg}$ II h-k doublet is fundamental for the correct modeling of the linear polarization produced by scattering processes in these lines (see also del Pino Alemán et al. 2016). In order to take into account this physical ingredient, we must solve the SE equations for the multi-term atom. The bottom-left panel of Fig. 3 shows the impact of the upper-term quantum interference on the shape of the broadband polarization pattern around the h-k resonances (solid curves) for two lines of sight with different values of $\mu=\cos \theta$, where $\theta$ is the heliocentric angle ${ }^{4}$ For comparison, we also show the $Q / I$ profile obtained by solving the SE equations for the multi-level atom, i.e., neglecting the upper-term quantum interference (dashed curves). With regard to the broadband $Q / I$ pattern across the h-k doublet, the differences between the multi-term and multi-level solutions are significant, and characteristic of the ${ }^{2} \mathrm{P}-{ }^{2} \mathrm{~S}$ resonant transitions.
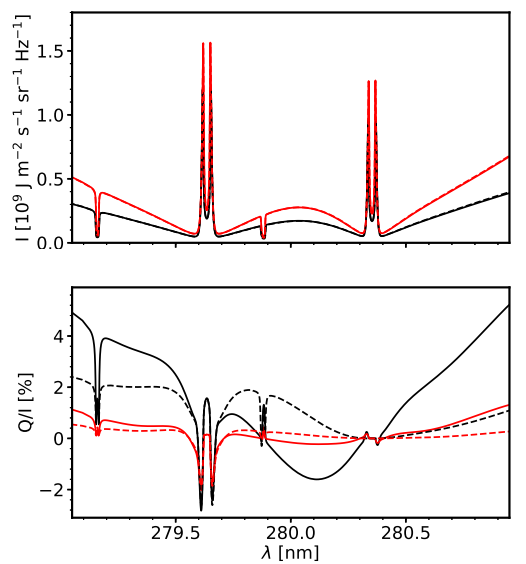
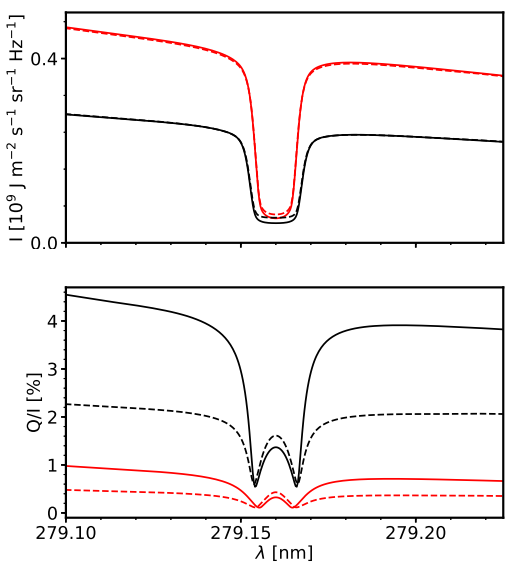
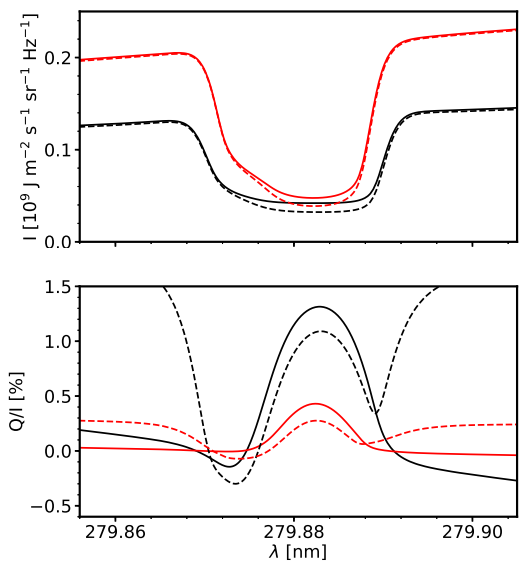

Fig. 3.- Profiles of Stokes $I$ (top row) and $Q / I$ (bottom row) of the Mg II h-k doublet and UV triplet in the FAL-C model. The first column shows the full spectral range, while the second and third columns show the spectral regions around the subordinate lines. The solid (dashed) curves represent the multi-term (multi-level) solution. The color of the curves indicates the $\mu$ value of the LOS: 0.1 (black) and 0.5 (red).

We point out that in our multi-term approach we assume that every transition pertaining to the same multiplet shares the same average radiation field (i.e., a "flat" spectrum over the frequency range spanned by the fine-structure transitions within a given multiplet). While this assumption ensures the internal consistency of the SE equations in the CRD limit (Landi Degl'Innocenti \& Landolfi 2004), it can have an impact on the emergent spectral profiles. The $\mathrm{Mg}$ II transitions in this study are all very close in wavelength (within $\sim 7 \AA$ ), and the spectral modulation of the emergent intensity profile is not significant enough to warrant relaxing the CRD approximation for the solution of the SE problem. Although there are measurable differences between the radiation field tensors of the $\mathrm{h}$ and $\mathrm{k}$ lines, those differences around the height of formation are not significant, and have a minimal impact on the line core of the emergent Stokes profiles. On the other hand, the impact of the flat-spectrum approximation is more significant for the subordinate lines, and the differences in the emergent linear polarization follow closely the behavior of the radiation anisotropy: $\mathrm{s}_{\mathrm{b}}$ has larger anisotropy and linear polarization, while $\mathrm{s}_{\mathrm{r}}$ shows less anisotropy and linear polarization.

Nevertheless, because the quantum interference between the $J$-levels of the ${ }^{2} \mathrm{P}$ term is a necessary physical ingredient to realistically model the linear polarization of the Mg II system, all the synthetic profiles shown in the rest of this paper have been computed using the multi-term model.

Figure 4 shows the intensity and fractional linear polarization profiles for the FAL-C (average atmosphere) and FAL-P (plage) semi-empirical models. Because these models are static and non-magnetic, all the differences between the two calculations are due to the thermodynamic structure of the model atmosphere, that is, how the atmospheric temperature and density change with height. The FAL-P emergent intensity is larger than its FAL-C counterpart across the whole spectral region. Such an increase in intensity is a consequence of the larger temperature (and consequently larger electron density) below the chromosphere-corona transition region in the case of the FAL-P model. The effect on the fractional linear polarization is the opposite, being smaller in the FAL-P model. In fact, FAL-P produces a lower degree of anisotropy in the subordinate lines than FAL-C (see Fig. 5), leading to a smaller degree of atomic alignment and, consequently, a smaller fractional linear polarization in the plage model.

\footnotetext{
${ }^{4}$ It is well known that, for symmetry reasons, the emergent polarization along the vertical direction of an axially symmetric $1 \mathrm{D}$ model cannot be linearly polarized. Larger heliocentric angles favor scattering polarization and thus we choose values of $\mu=0.1$ and 0.5 in our figures
} 

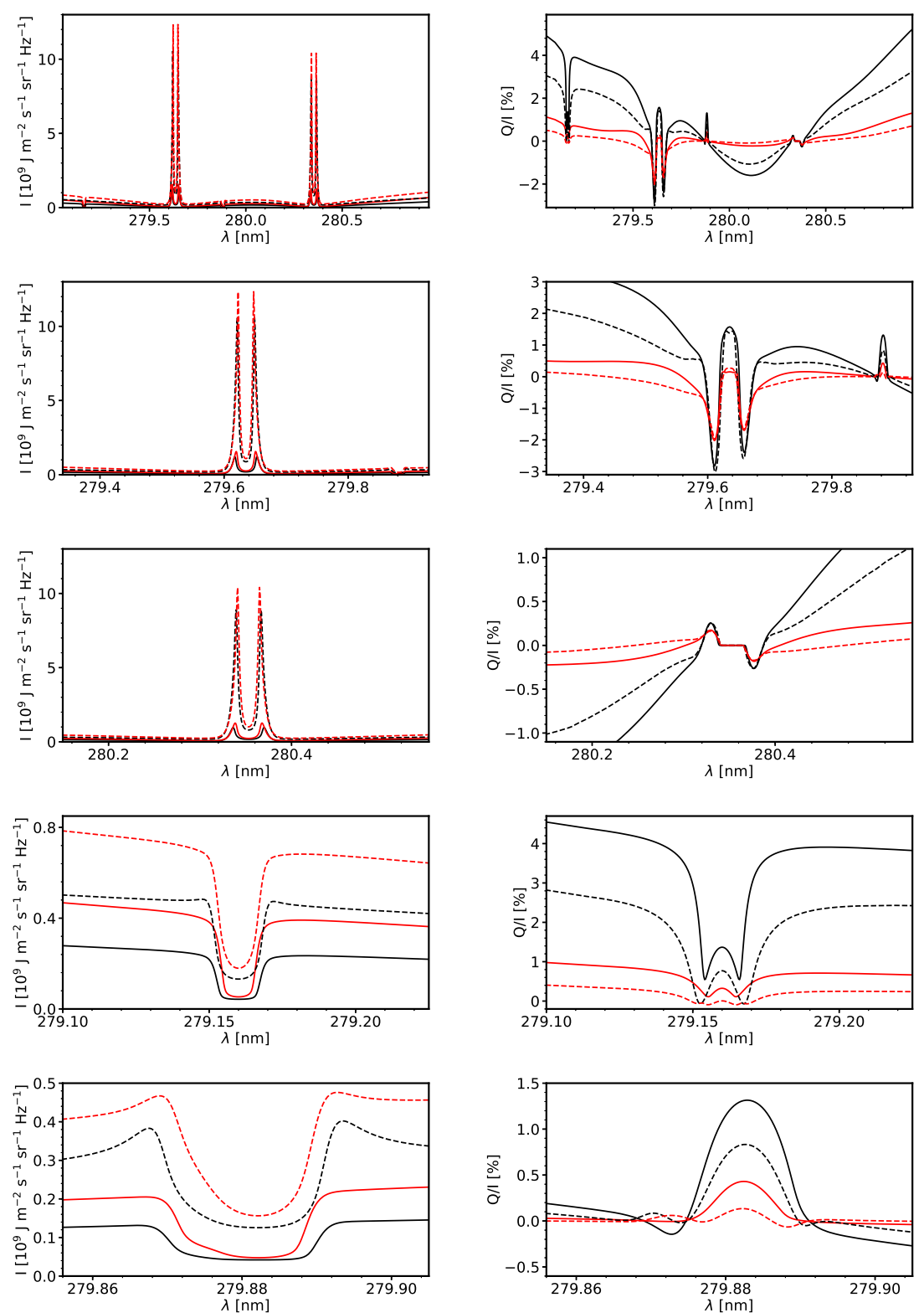

Fig. 4.- Profiles of Stokes $I$ (left column) and $Q / I$ (right column) of the Mg II h-k doublet and UV triplet. The first row shows the full spectral range, while the other rows show, in order, the spectral details of $\mathrm{k}, \mathrm{h}, \mathrm{s}_{\mathrm{b}}$, and $\mathrm{s}_{\mathrm{r}_{\mathrm{a}}}+\mathrm{s}_{\mathrm{r}_{\mathrm{b}}}$. The solid (dashed) curves correspond to the calculations using the FAL-C (FAL-P) model atmosphere. The color of the curves indicates the $\mu$ value of the LOS: 0.1 (black) and 0.5 (red).

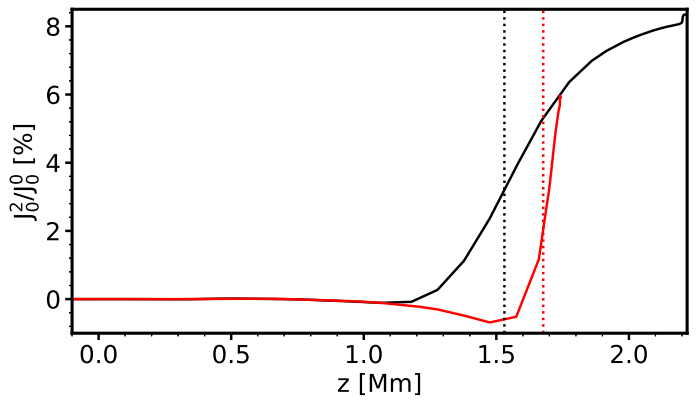

FIG. 5. - Variation with height of the fractional anisotropy $J_{0}^{2} / J_{0}^{0}$ for the Mg II UV triplet in the FAL-C (black) and FAL-P (red) models. The vertical dotted lines show the largest height where the optical depth is unity within the range of the subordinate lines for a LOS with $\mu=0.1$ 


\subsection{Magnetic Sensitivity}

In this section we add a magnetic field of given strength and orientation to the FAL-C and FAL-P atmospheric models in order to study the magnetic sensitivity of the Mg II h-k doublet and UV triplet. Figures 6 and 7 show the emergent fractional linear polarization along a LOS with $\mu=0.1$ for a horizontal magnetic field with strengths of 0 , $10,20,50,100$, and $200 \mathrm{G}$. For these magnetic field strengths, we see two physical processes shaping the polarization profiles. At the line center of the $\mathrm{k}$ and subordinate lines, the magnetic field relaxes the coherence among the magnetic sublevels (Hanle effect) leading to a depolarization of the signal with respect to the zero-field case. Outside the atomic resonances, the depolarization of the $Q / I$ profile and the corresponding appearance of a Stokes $U / I$ signal are due to $\mathrm{M}-\mathrm{O}$ effects in the line wings. These effects couple the $Q$ and $U$ Stokes parameters, and transfer the broadband polarization, created by PRD in a magnetically split line, from one Stokes parameter to the other. At the same time, the total linear polarization $\sqrt{Q^{2}+U^{2}}$ is also reduced due to radiation transfer effects (del Pino Alemán et al. 2016. Alsina Ballester et al. 2016).

None of the levels of the $\mathrm{h}$ transition can carry atomic alignment and, therefore, the line core does not show scattering polarization and is thus unaffected by the Hanle effect. The transition $\mathrm{s}_{\mathrm{r}_{\mathrm{a}}}$ shows a very small linear polarization signal, which is practically of no diagnostic use. Regarding the M-O effects in the line wings, they display a magnetic sensitivity similar to that of the $\mathrm{k}$ line (the broadband pattern is due entirely to the combined action of PRD effects and quantum interference in the upper term of the h-k doublet). Consequently, the amplitude of the broadband polarization vanishes for magnetic fields in the saturation regime of the $\mathrm{k}$ line (fields larger than $50 \mathrm{G}$ in Figs. 6 and 7).

The polarization signal at the core of the $\mathrm{k}$ line shows quantitatively a very similar behavior in both the FAL-C (Fig. 6) and FAL-P (Fig. 7) models. This happens because the core of this transition forms in the top layers of the atmosphere, where the two models are relatively similar. On the other hand, the polarization of the broadband profile comes from an extended layer in the upper photosphere (with its maximum response around $\sim 500 \mathrm{~km}$ above the visible surface), while the cores of the subordinate lines form at chromospheric heights, with the polarization signal being sensitive to the presence of magnetic fields in a relatively extended layer above the line core height of formation. Note that the FAL-P model shows a significant compression in height in comparison to the FAL-C model, and thus the subordinate and resonance lines form much closer in geometric scale in the plage model than in the FAL-C average atmosphere. This can be more easily seen in Fig. 8, which shows the response function of the Stokes-Q and Stokes- $U$ parameters to perturbations in the magnetic field. The response function tells us how the emergent Stokes signal responds to a change in a physical parameter (e.g., the magnetic field) at each point within the model atmosphere and for each wavelength (Landi Degl'Innocenti \& Landi Degl'Innocenti 1977). Because the two atmospheric models are different everywhere except in the lower photosphere and at the very top layers, the quantitative behavior of the fractional linear polarization differs in the whole spectral range except at the core of the $\mathrm{k}$ line.

The second column in Figs. 6 and 7 shows the fractional linear polarization profile $U / I$. Because in a $1 D$ axially symmetric atmosphere the $U / I$ polarization is zero in the absence of fields, the introduction of a magnetic field inclined with respect to the local vertical is necessary to generate Stokes $U$. At the same time, the field has overall a depolarizing effect, because of the relaxation of the quantum coherence of the atomic levels (Hanle effect). Therefore, for magnetic fields below the critical Hanle field strength $\left(\sim 20,60,10\right.$, and $40 \mathrm{G}$ for $\mathrm{k}, \mathrm{s}_{\mathrm{b}}, \mathrm{s}_{\mathrm{r}_{\mathrm{a}}}$, and $\mathrm{s}_{\mathrm{r}_{\mathrm{b}}}$, respectively), the $U / I$ polarization amplitude increases with the magnetic strength (at the expense of the $Q / I$ polarization). Above the critical Hanle field strength, the magnetic field reduces both the $Q / I$ and $U / I$ polarization signals due to the Hanle effects. It is important to emphasize that, in a 3D model atmosphere without axial-symmetry constraint, a non-zero $U / I$ profile can emerge even in the absence of magnetic fields (e.g., Štěpán \& Trujillo Bueno 2016). In such a case, the presence of a magnetic field typically results in a reduction of the zero-field polarization signal (see also del Pino Alemán et al. 2018).

Figure 9 shows the $Q / I$ polarization signal as a function of magnetic strength, for a homogeneous magnetic field with different inclinations, and after integrating the signal over all possible magnetic field azimuths. This mimics the behavior of a "canopy" field taking all possible azimuth orientations within the spatial resolution element. From top to bottom, the various panels give the polarization at the line center of the $\mathrm{Mg}$ II transitions (with the exception of $\mathrm{h}$, which is unpolarized), and at the wavelength $280.1 \mathrm{~nm}$, in the continuum between $\mathrm{h}$ and $\mathrm{k}$, which shows the most negative polarization. The first conclusion we can draw is that the behavior of the polarization signal with the magnetic field strength and inclination is qualitatively the same in both the FAL-C (left panels) and FAL-P (right panels) models, with the only difference that the polarization emerging from the FAL-P model is consistently smaller. Therefore, we expect smaller polarization signals in plage regions, even before taking into account that the stronger magnetic fields of plages are already more effective in depolarizing the scattered radiation than in the quiet Sun. The only wavelength where the two models do not show the same qualitative behavior is for the $\mathrm{s}_{\mathrm{r}_{\mathrm{a}}}$ line center. However, its polarization signal is so small that this line is of no interest for magnetic field diagnostics and thus we do not include a figure for this wavelength.

As it is expected from the theory, because the line center is dominated by scattering polarization and the Hanle effect, the sensitivity to vertical (black curves) or quasi-vertical (10 ${ }^{\circ}$ inclination, red curves) magnetic fields is quite small, while the depolarization is maximum for inclinations close to the Van Vleck angle $\left(\sim 54.7^{\circ}\right.$; green curves). On the other hand, the polarization outside the line cores (e.g., last row in Fig. 9 is due to PRD effects, quantum interference, and to the M-O effects caused by the Zeeman splitting. Therefore, the behavior of the polarization signal with the magnetic field strength for different inclinations is also qualitatively different than for the line centers.

Because we averaged over all possible magnetic field azimuths, for symmetry reasons the $U / I$ signals in the line cores 

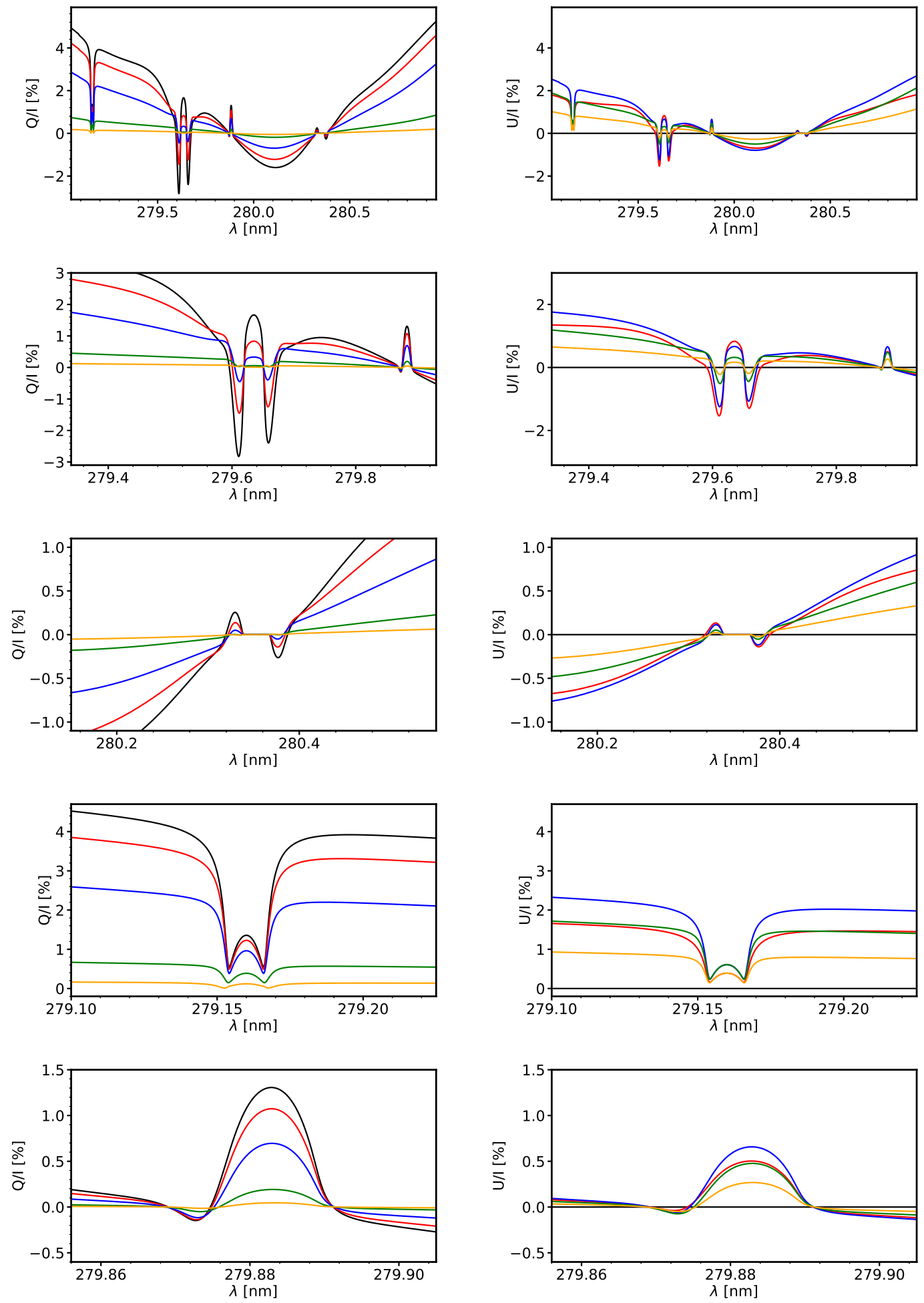

FIG. 6. - Fractional linear polarization profiles $Q / I$ (left column) and $U / I$ (right column) for the Mg II h-k doublet and UV triplet in the FAL-C model, for a LOS with $\mu=0.1$. The first row shows the full spectral range, while the second to fifth rows show the spectral regions around the transition lines. The color of the curves indicates the strength of the horizontal magnetic field pointing towards the observer: $0 \mathrm{G}$ (black), $10 \mathrm{G}$ (red), $20 \mathrm{G}$ (blue), $50 \mathrm{G}$ (green), and $100 \mathrm{G}$ (orange). 

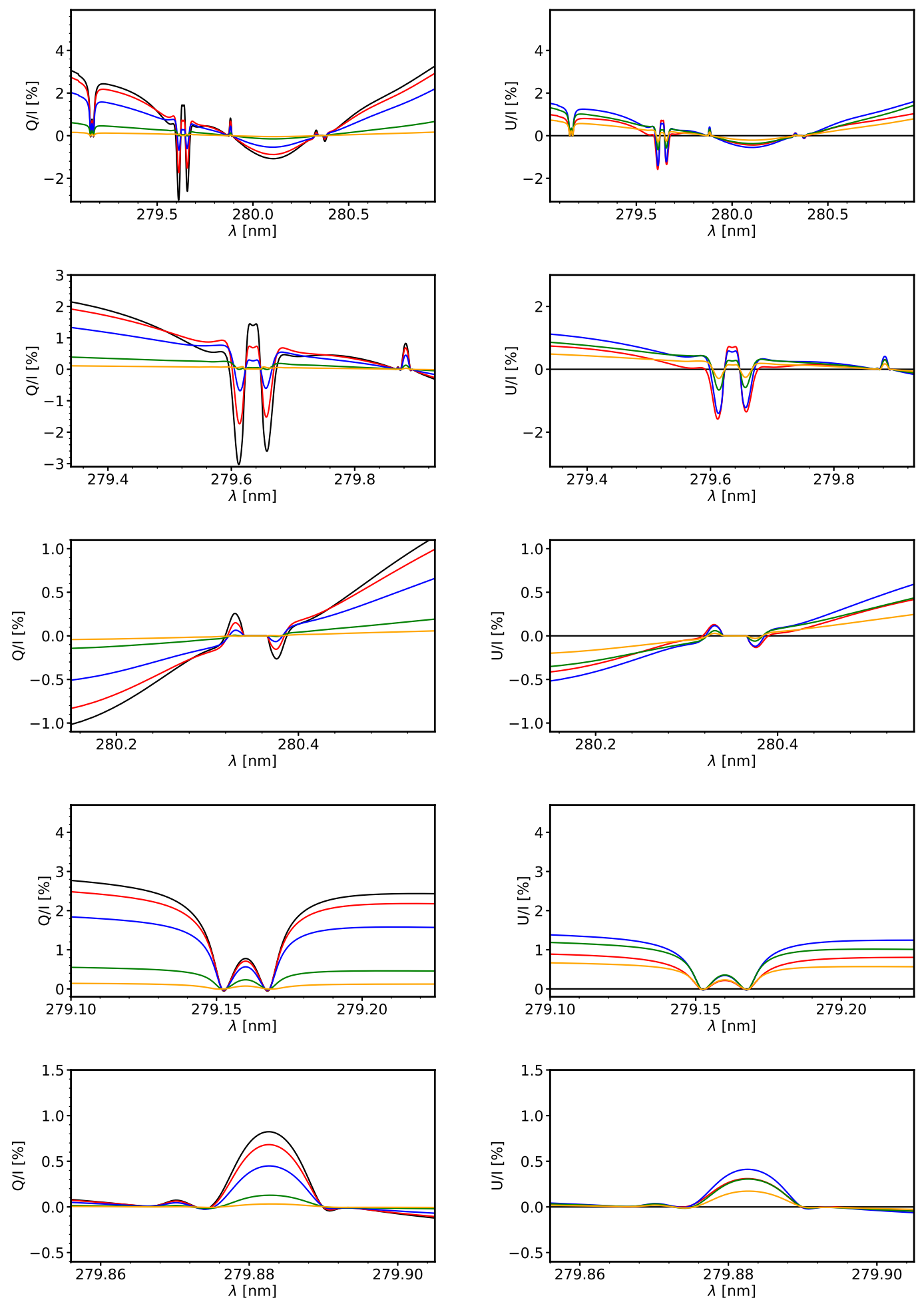

FIG. 7.- Like in figure 6 but for the FAL-P model.

due to the Hanle effect cancel out. However, this is not the case for the $U / I$ broadband polarization. Fig. 10 shows the fractional linear polarization $U / I$ at the continuum wavelength $280.1 \mathrm{~nm}$ for the same models of Fig. 9 . Notably, vertical fields generate the largest $U / I$ signals. Initially, increasing the magnetic field strength results in a larger $U / I$ polarization signal, as more $Q / I$ polarization is being "rotated" into $U / I$. This trend of $U / I$ polarization has a turning point (dependent on the field inclination), as a consequence of the overall depolarization induced by the M-O effects in an optically thick atmosphere (see also Alsina Ballester et al. 2018).

Because the broadband pattern of polarization depends on both the magnetic field and the 3D thermal structure 

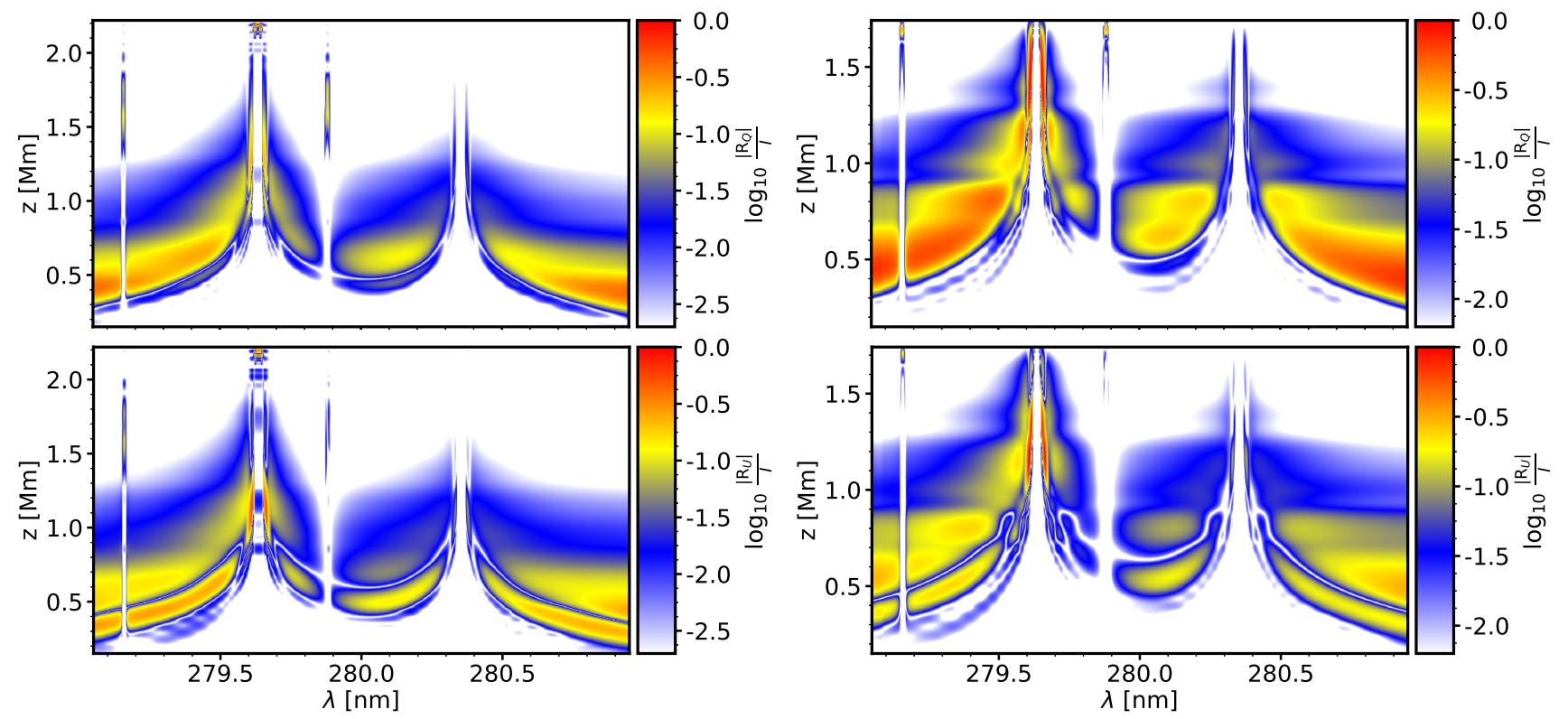

FIG. 8.- Absolute value of the Stokes-Q (top row) and Stokes-U (bottom row) response functions for the Mg II h-k doublet and the UV triplet in the FAL-C (left) and FAL-P (right) models for a LOS with $\mu=0.1$. The reference model has a horizontal magnetic field of 20G directed towards the observer and it is perturbed with a horizontal magnetic field of $1 \mathrm{G}$, pointing in the same direction.
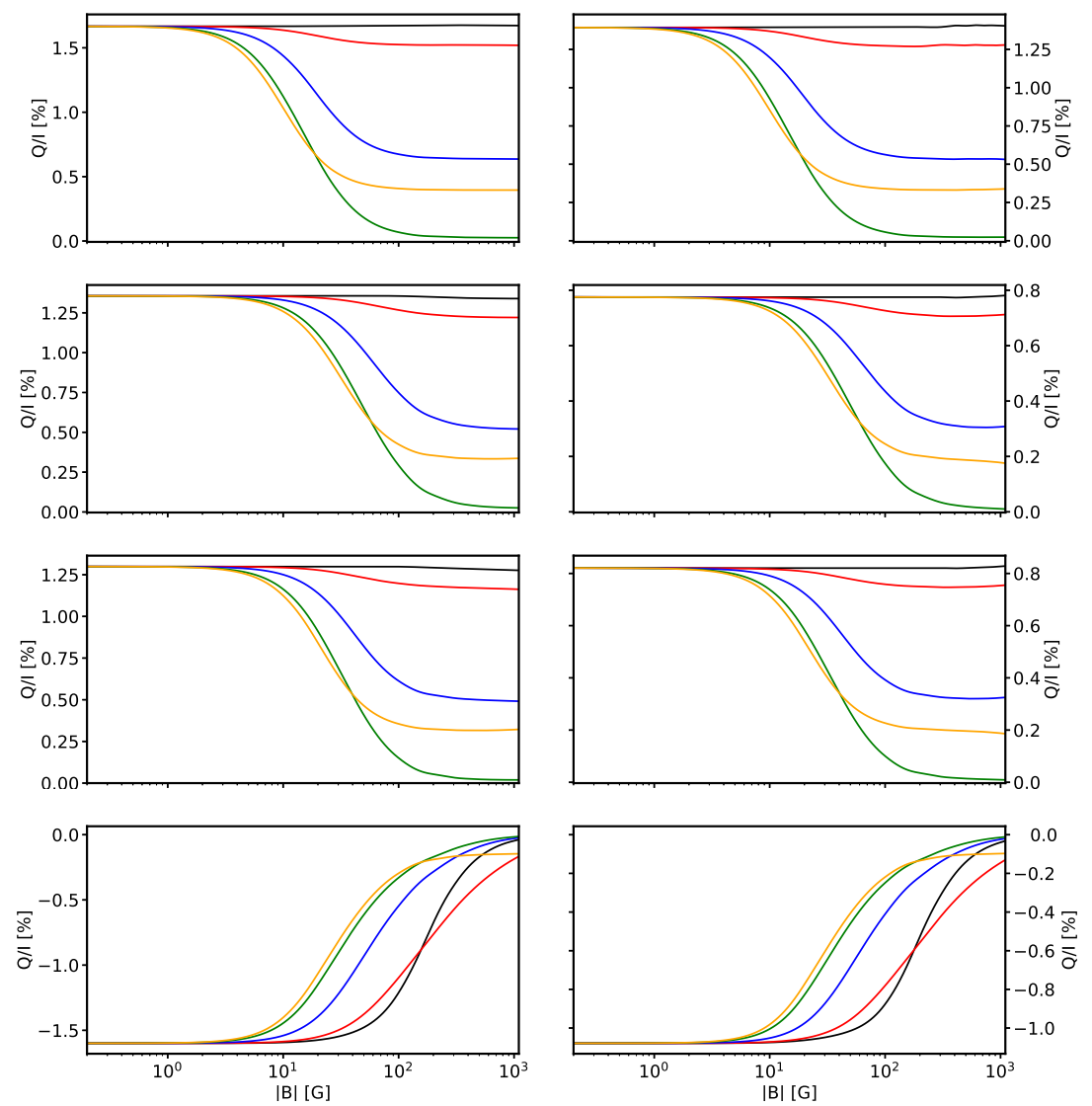

FIG. 9. - Variation of the fractional linear polarization $Q / I$ with the magnetic field strength for a homogeneous magnetic field with a fixed inclination, averaged over all possible azimuths of the magnetic field, for a LOS with $\mu=0.1$. The left (right) column shows calculations using the FAL-C (FAL-P) model. From top to bottom, each row shows the polarization at the center of the $\mathrm{k}, \mathrm{s}_{\mathrm{b}}$, $\mathrm{s}_{\mathrm{b}}$ lines, and at a wavelength $(280.1 \mathrm{~nm})$ close to the minimum of the broadband pattern between $\mathrm{h}$ and $\mathrm{k}$. The colors correspond to different inclinations of the magnetic field: $0^{\circ}$ (black), $10^{\circ}$ (red), $30^{\circ}$ (blue), $60^{\circ}$ (green), and $90^{\circ}$ (orange). 

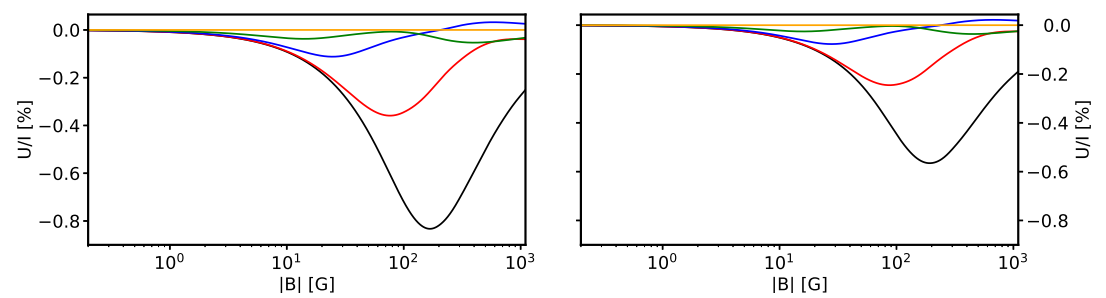

FIG. 10.- Variation of the fractional linear polarization $U / I$ with the magnetic field strength for a homogeneous magnetic field with a fixed inclination, averaged over all possible azimuths, for a LOS with $\mu=0.1$, at a wavelength $(280.1 \mathrm{~nm})$ close to the minimum of the broadband pattern between $\mathrm{h}$ and $\mathrm{k}$. The left (right) column shows calculations using the FAL-C (FAL-P) model. The colors correspond to different inclinations of the magnetic field: 0 (black), $10^{\circ}$ (red), $30^{\circ}$ (blue), $60^{\circ}$ (green), and $90^{\circ}$ (orange).

of the solar atmosphere, it can be difficult to unequivocally demonstrate the manifestation of M-O effects through an observing sequence of only a few minutes. Nevertheless, we know that a very strong magnetic field should be able to completely destroy this broadband polarization. Therefore, spectropolarimetric observations where the slit stretches over both the quiet Sun and a strong active region would be helpful to establish the actual role of M-O effects: if the broadband polarization pattern were visible in the quiet-Sun region but disappeared in the strong-field region, this could only be due to M-O effects, since it is the only mechanism that can completely destroy linear polarization ${ }^{5}$
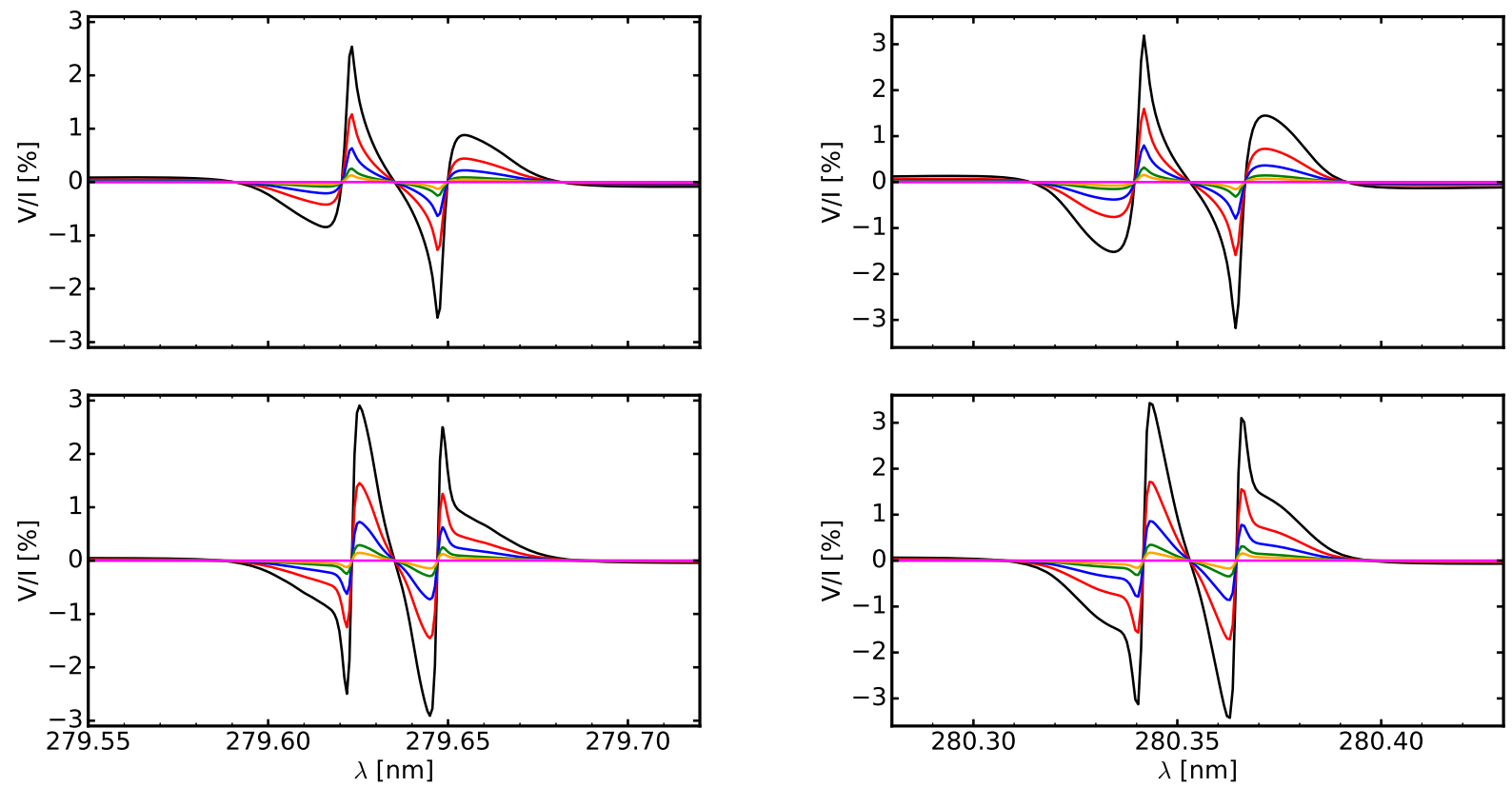

FIG. 11. - Fractional circular polarization profile $V / I$ for the $\mathrm{Mg}$ II h (right column) and $\mathrm{k}$ (left column) doublet. The top (bottom) row shows calculations using the FAL-C (FAL-P) model, for a LOS with $\mu=1.0$. The color of the curves indicates the strength of the vertical magnetic field: $0 \mathrm{G}$ (magenta), $10 \mathrm{G}$ (orange), $20 \mathrm{G}$ (green), $50 \mathrm{G}$ (blue), $100 \mathrm{G}$ (red), and $200 \mathrm{G}$ (black).

Finally, Figs. 11 and 12 show the fractional circular polarization profile, Stokes $V / I$, for vertical magnetic fields with different strengths and a LOS pointing at disk center. In this geometry, only circular polarization is possible in a 1D axially symmetric model atmosphere. While the subordinate lines are less polarized in the plage model, the resonance lines show a similar degree of polarization in the FAL-C and FAL-P models with regard to the inner lobes of the profile (closer to line center). The outer lobes of the $V / I$ polarization are due to PRD effects, and are significantly larger in the plage model, likely a consequence of the smaller geometrical extension of the model atmosphere. Due to this "compression" of the plage model, the outer peaks respond more strongly to magnetic fields from $\sim 1500 \mathrm{~km}$ upwards (see Fig. 13 right panel), quite close to the region where the inner lobes form. The farther, smoother part of the profile forms in a much more extended region. In the average FAL-C atmospheric model, instead, the response is more extended in height, resulting in a smoother $V / I$ profile.

\subsection{Dynamic Sensitivity}

In this section we study the sensitivity of the Mg II h-k and UV triplet lines to vertical velocity gradients, and the effect of the time integration on the observed Stokes profiles. In order to achieve this, we solve the polarized radiation

${ }^{5}$ Other physical mechanisms, such as a significant increase of collisional rates, can also completely destroy linear polarization, but there is no physical reason why these rates should be much larger in an active region than in the surrounding quiet-Sun atmosphere. 

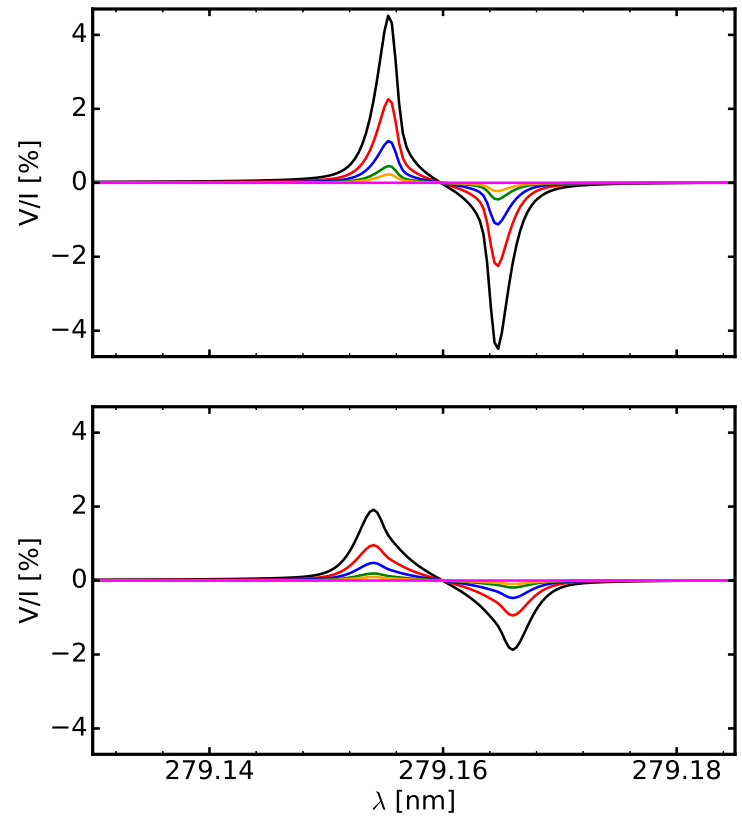
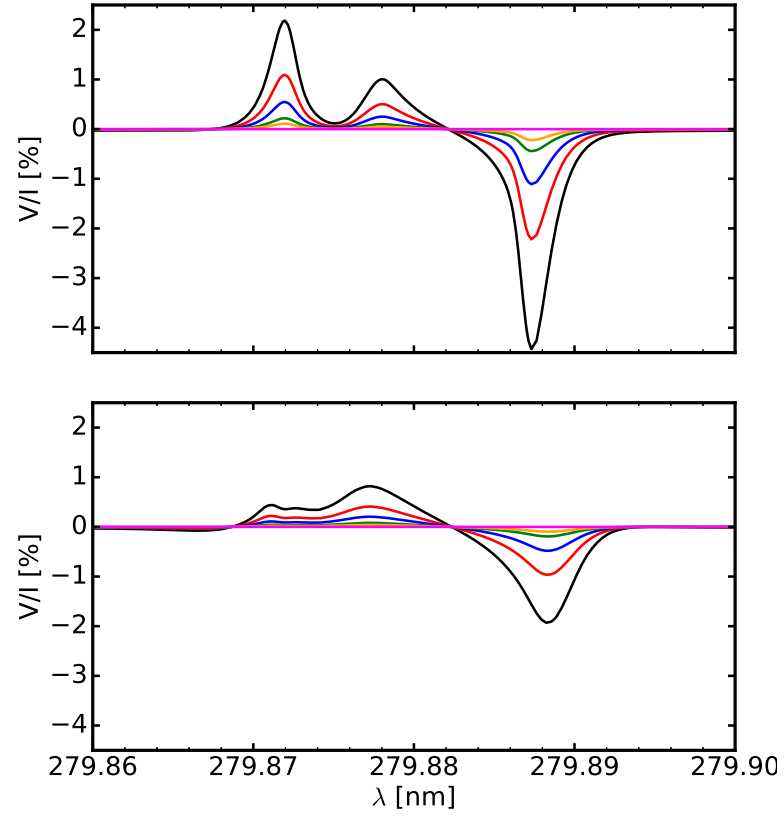

FIG. 12. - Fractional circular polarization profile $V / I$ for the Mg II UV triplet. The left (right) column shows the spectral range around $\mathrm{s}_{\mathrm{b}}$ $\left(\mathrm{s}_{\mathrm{r}}\right)$. The top (bottom) row shows calculations using the FAL-C (FAL-P) model, for a LOS with $\mu=1.0$. The color of the curves indicates the strength of the vertical magnetic field: $0 \mathrm{G}$ (magenta), $10 \mathrm{G}$ (orange), $20 \mathrm{G}$ (green), $50 \mathrm{G}$ (blue), $100 \mathrm{G}$ (red), and $200 \mathrm{G}$ (black).
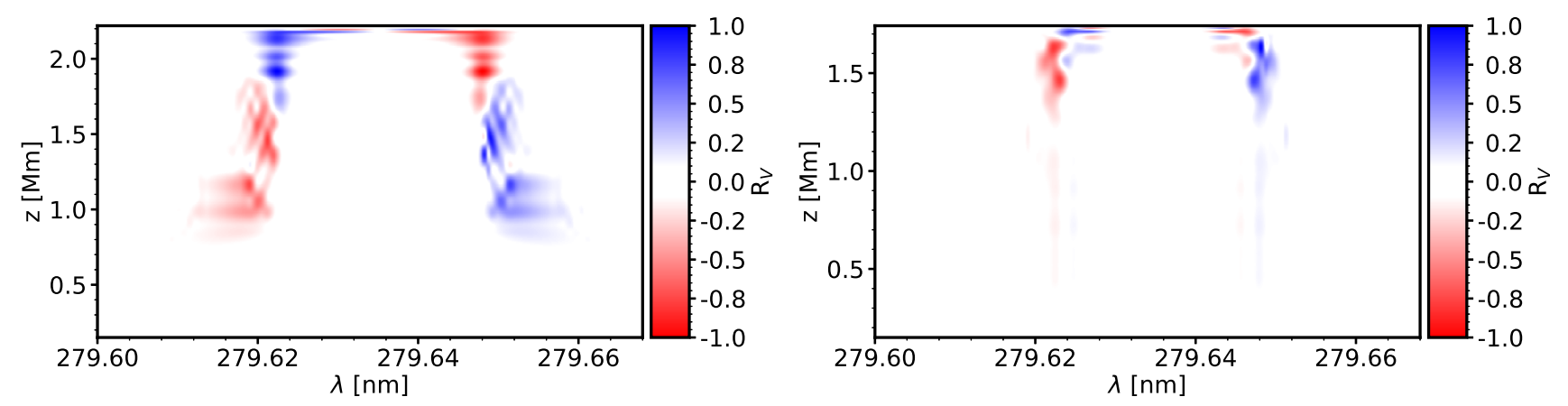

FIG. 13.- Normalized value of the Stokes $V$ response function to magnetic field perturbations for the Mg II k line in the FAL-C (left) and FAL-P (right) models, for the disk-center LOS. The calculation considers a reference model atmosphere with a vertical magnetic field of $200 \mathrm{G}$, pointing towards the observer, and a perturbation of $1 \mathrm{G}$.

transfer problem at each time step in the CS time-dependent hydrodynamic model (Carlsson \& Stein 1997). Our calculations of the angle-dependent PRD have been carried out with the observer's frame method using a suficiently large number of frequency nodes (of the order of $10^{3}$ ). The presence of velocity fields introduces changes in the radiation anisotropy because of the associated Doppler shifts of the spectral line radiation. Hence, we must solve the problem using the more general angle-dependent redistribution function for coherent scattering. However, as the velocity in this $1 \mathrm{D}$ time series is always directed along the vertical, we can still take advantage of the axial symmetry of the model in order to simplify the calculation 6

Figure 14 shows some of the main characteristics of the formation of the Mg II lines in the CS model. The heights where the optical depth is unity (a rough estimation of the height of formation) for the resonance and the subordinate lines are in opposition of phase (Fig. 14, top panel). The lower-boundary piston used by Carlsson \& Stein (1997) to drive the hydrodynamic simulation makes the atmosphere oscillate in such a way that it goes through compression and expansion phases. Given that the resonance lines form higher in the atmosphere and the subordinate lines are formed at much lower heights, the regions of formation get closer during the compression phase and separate instead during the expansion phase.

During compression, a temperature shock rises through the atmosphere (see Carlsson \& Stein 1997), producing a much hotter temperature minimum (Fig. 14 second panel, black curve). The red curve in the second panel of Fig. 14 shows the height with the maximum temperature gradient, which traces the position of the transition region. The blue curve in the same panel shows the height of the temperature minimum. During a compression phase, the CS

${ }^{6}$ For general, non-axially symmetric problems, we would need to take into account at least a factor 8 more directions, and even more in order to attain sufficient accuracy in the polarization profiles. 


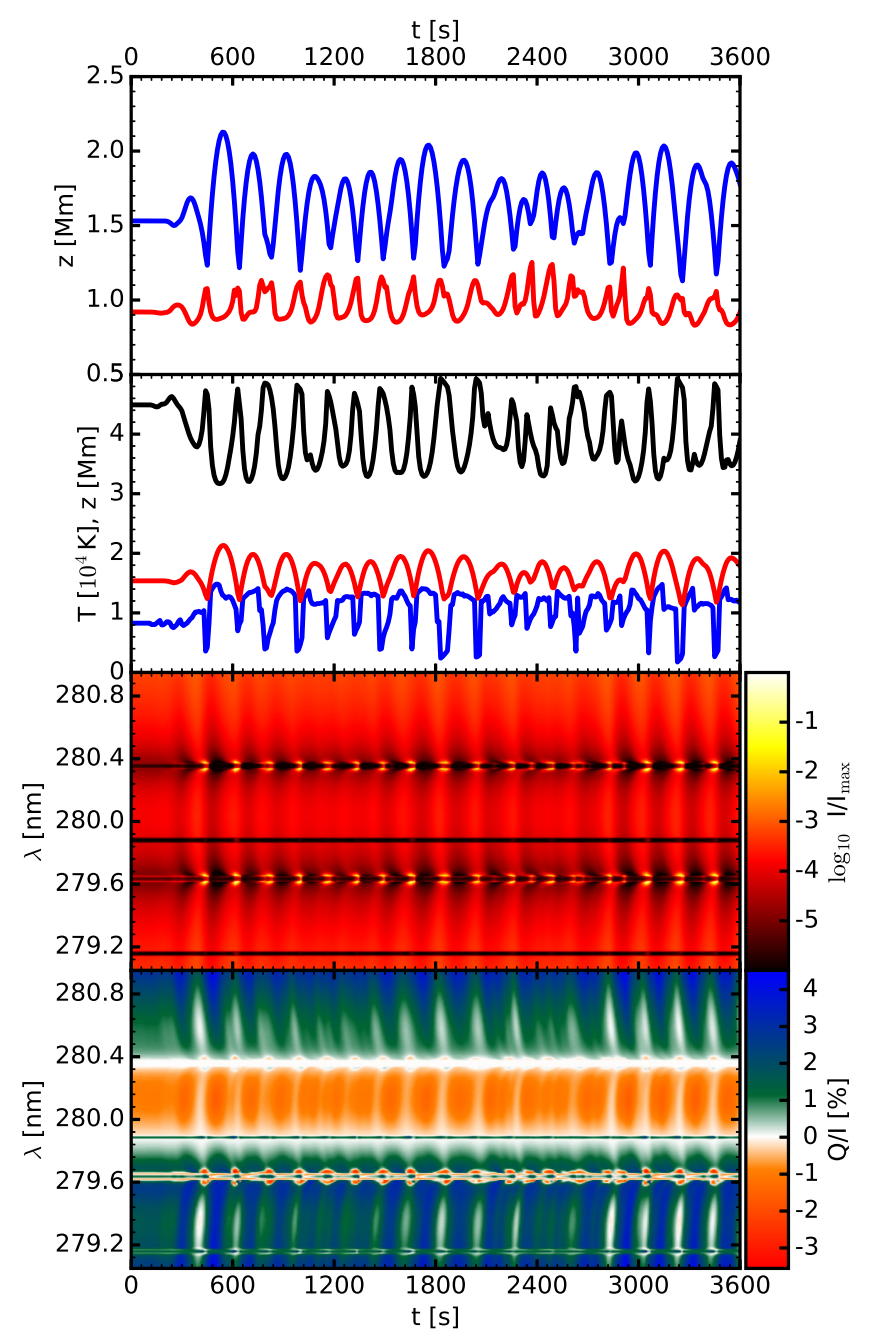

FIG. 14.- Top panel: variation with time of the height where the optical depth is equal unity for the line center of the k line at 279.64 $\mathrm{nm}$ (blue) and of the $\mathrm{s}_{\mathrm{r}}$ transition at $279.88 \mathrm{~nm}$ (red). Second panel: variation with time of the temperature minimum (black), the height corresponding to the temperature minimum (blue), and the height with the maximum temperature gradient (red). Third panel: variation with time of the intensity profile around the Mg II resonance lines, normalized to the maximum value in the range. Bottom panel: variation with time of the fractional polarization $Q / I$ profile around the resonance lines of $\mathrm{Mg}$ II. The scale is saturated to $|Q / I|=2$ to facilitate the visualization, but the polarization in this range reaches values of $4 \%$.

atmosphere has a different stratification from a typical semi-empirical model atmosphere (color curves in Fig. 2 top-left panel) due to the shock. During an expansion phase, a "valley" with an evident temperature minimum is created in the space left by the rising transition region and the falling photosphere.

The temporal change of the heights of formation is due to the change of the thermal structure of the CS model, and not directly to the presence of velocity gradients. In fact, when we solve the radiation transfer problem excluding velocities, we obtain very similar results for those heights. This is not the case for the radiation field and the emergent Stokes parameters. Fig. 15 shows the variation with height and time of the fractional anisotropy for the Mg II h-k doublet and UV triplet, excluding (left) and including (right) the velocity field in the solution of the radiation transfer problem. In agreement with previous studies of different spectral lines (e.g., Carlin et al. 2012 Sampoorna \& Nagendra 2015), the anisotropy is significantly enhanced in the presence of a velocity field with gradients. In particular, at some time steps, the anisotropies of the resonance and subordinate lines appear to be enhanced by a factor $\sim 2$ and $\sim 4$, respectively. This is more directly seen in Fig. 16, where we show the average anisotropy in the CS time series including (blue curve) and excluding (red curve) velocities, together with the corresponding full ranges of values (shaded areas), as a function of height. For comparison we also show in Fig. 16 the anisotropy in the unperturbed FAL-C (black curve) and FAL-P (green curve) models. Because the "absolute" height of formation is changing continuosly with time, we set the zero of the height axis to the maximum height of optical depth unity, separately for each set of transitions and atmospheric model. The anisotropy reaches the largest values in the presence of velocity gradients (blue shaded areas). This enhacement is much more significant in the subordinate lines (left panel of Fig. 16) and their anisotropy, averaged over the whole series, is enhanced in the presence of velocity gradients (the result is similar when taking the median). The anisotropy in the resonance lines (right panel of Fig. 16) also changes notably between including and excluding velocity gradients during the time series, but the differences in the average anisotropy is not significant. 

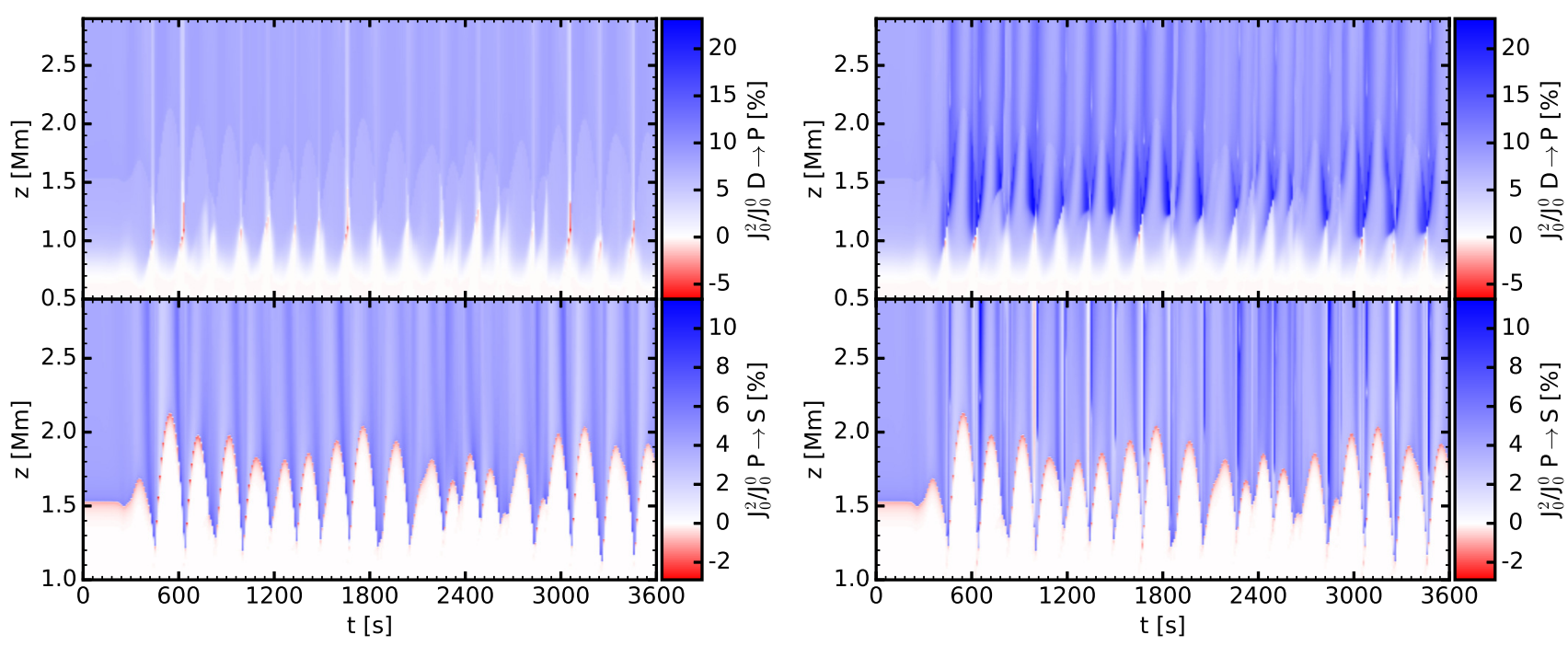

FIG. 15.- Variation with time and height of the fractional anisotropy $J_{0}^{2} / J_{0}^{0}$ for the $\mathrm{Mg}$ II UV triplet (top panels) and the Mg II h-k doublet (bottom panels) in the CS time series. The left (right) panels correspond to the solution of the radiation transfer problem when excluding (including) the presence of the velocity fields of the model atmosphere.
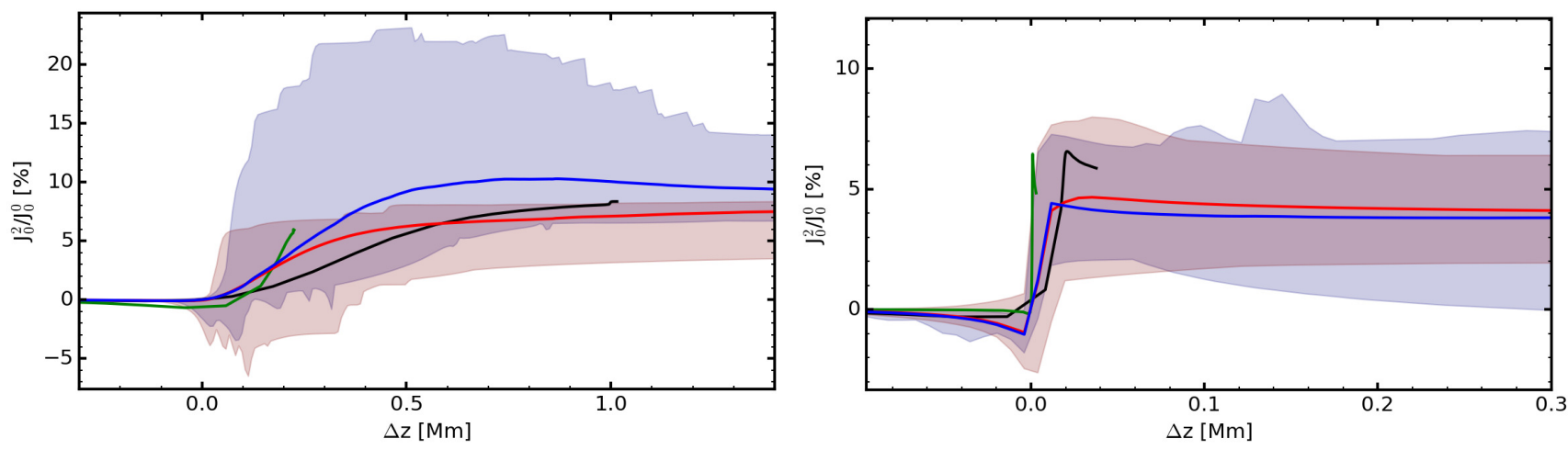

FIG. 16. - Variation with height of the fractional anisotropy $J_{0}^{2} / J_{0}^{0}$ for the Mg II UV triplet (left panel) and the Mg II h-k doublet (right panel). The shaded areas demarcate the range of values of the anisotropy attained during the CS time series, including (light blue) and excluding (light red) velocities. The solid curves of the corresponding colors show the average anisotropy over the time series for the two cases. For comparison, the black (green) curve shows the anisotropy in the FAL-C (FAL-P) atmospheric model. We set the zero of the height axis at the maximum height of optical depth unity, within the wavelengths of the set of transitions for each model atmosphere.

Figure 17 shows the intensity and fractional polarization profiles corresponding to the FAL-C model and the time average over the full CS time series, with and without velocities. The subordinate lines are much wider in the CS series than in the FAL-C model. This is mainly due to our choice of microturbulent velocity. While $7 \mathrm{~km} / \mathrm{s}$ appears to be adequate to produce a profile width for the h-k doublet similar to that of the FAL-C model, it turns out to be excessive for the triplet. However, since the objective of this section is to study the impact of the velocity field, it is not necessary to determine the best microturbulence stratification (a very computationally intensive task), and it is sufficient to simply compare the emerging profiles in the CS series when velocities are included or excluded. While the impact of the velocity fields is small on the intensity profile of the subordinate lines, the fractional linear polarization clearly shows the effect of the anisotropy enhacement, resulting in a significantly larger polarization signal.

The situation changes dramatically for the h-k doublet. First, there is a clear asymmetry in the emission peaks of Stokes $I$ for both lines of the doublet, when velocities are included. The same asymmetry is also found in the fractional polarization profile (see Fig. 17). In fact, the degree of polarization is reduced, and the asymmetry due to the intrinsic physical properties of the $\mathrm{k}$ line (blue lobe larger than the red lobe) is reversed by the dynamics (i.e., the red lobe is now larger than the blue lobe). The polarization of these lines is clearly sensitive to the velocity field within the atmosphere.

The profiles shown in Fig. 17 are the result of the integration over the full series, i.e., 3600 s. However, in a real observation the integration time is significantly smaller. Fig. 18 shows the fractional polarization profile, at different times, for different exposure times. As expected, with small integration times (top panels), the profiles are quite different depending on the interval of the series that is being integrated (see Carlin et al. 2013). For larger integration times, the different profiles converge to each other and to the total average profile, as expected based on the behavior of the CS model. However, it is important to note that the fractional polarization of the spectral region between the $\mathrm{s}_{\mathrm{r}}$ and $\mathrm{h}$ lines "converges" in amplitude already for $60 \mathrm{~s}$ of exposure time, and the integrated signal is around a factor 

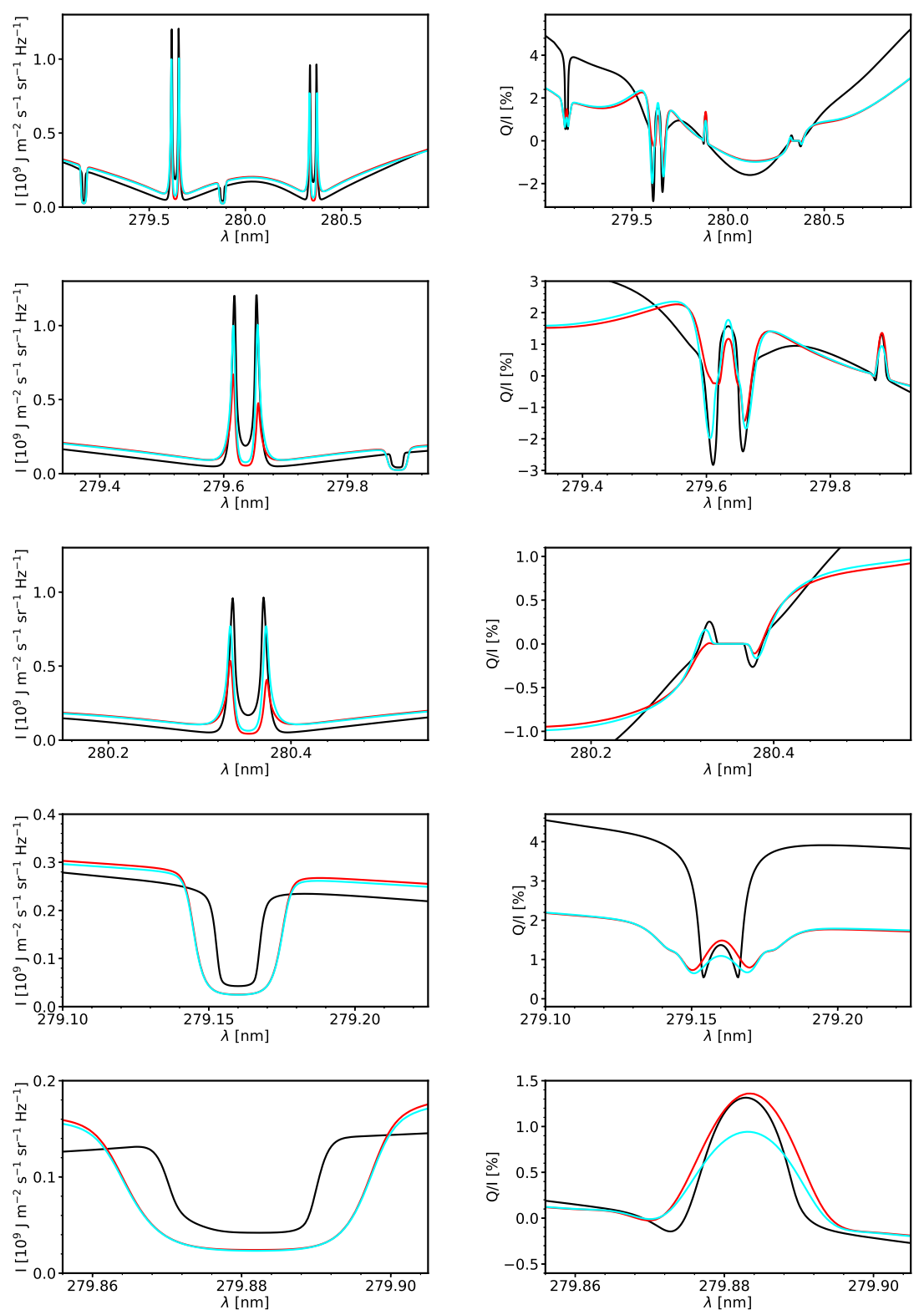

Fig. 17.- Intensity $I$ profiles (left column), and fractional linear polarization $Q / I$ profiles (right column) for the Mg II h-k doublet and UV triplet. The first row shows the full spectral range, while from the second to the fifth rows show the spectral regions around the individual transition lines. The black, light blue, and red curves correspond to the FAL-C, CS (ignoring velocity fields), and CS models, respectively, for a LOS with $\mu=0.1$.

2 smaller than the one expected from the calculations in the FAL-C model. Therefore, the joint effect of velocity and magnetic fields could make a clear detection of the negative fractional polarization in this spectral region more difficult.

\section{CONCLUSIONS}

We carried out a detailed numerical investigation of the intensity and polarization of the $\mathrm{Mg}$ II h-k doublet and UV triplet located around $280 \mathrm{~nm}$. We used radiative transfer calculations in 1D models of quiet and plage regions of the solar atmosphere, in order to study the sensitivity of the emergent Stokes profiles to the thermal, magnetic, and dynamic properties of those model atmospheres. These calculations take into account the combined action of anisotropic irradiation of the atomic system (with partial frequency redistribution for the h-k doublet) and the Hanle, Zeeman, and magneto-optical (M-O) effects, allowing us to model the presence of magnetic fields of arbitrary field strengths (i.e., in the general regime of the incomplete Paschen-Back effect).

We find that this UV spectral region is sensitive to the magnetic field over a wide range of heights. The core of $\mathrm{Mg}$ II $\mathrm{k}$ forms in the upper chromosphere, the subordinate lines and the near wings of $\mathrm{h}$ and $\mathrm{k}$ form in the intermediate chromosphere, whereas the broadband polarization pattern of the h-k wings is sensitive to conditions in the upper photosphere and low chromosphere. 


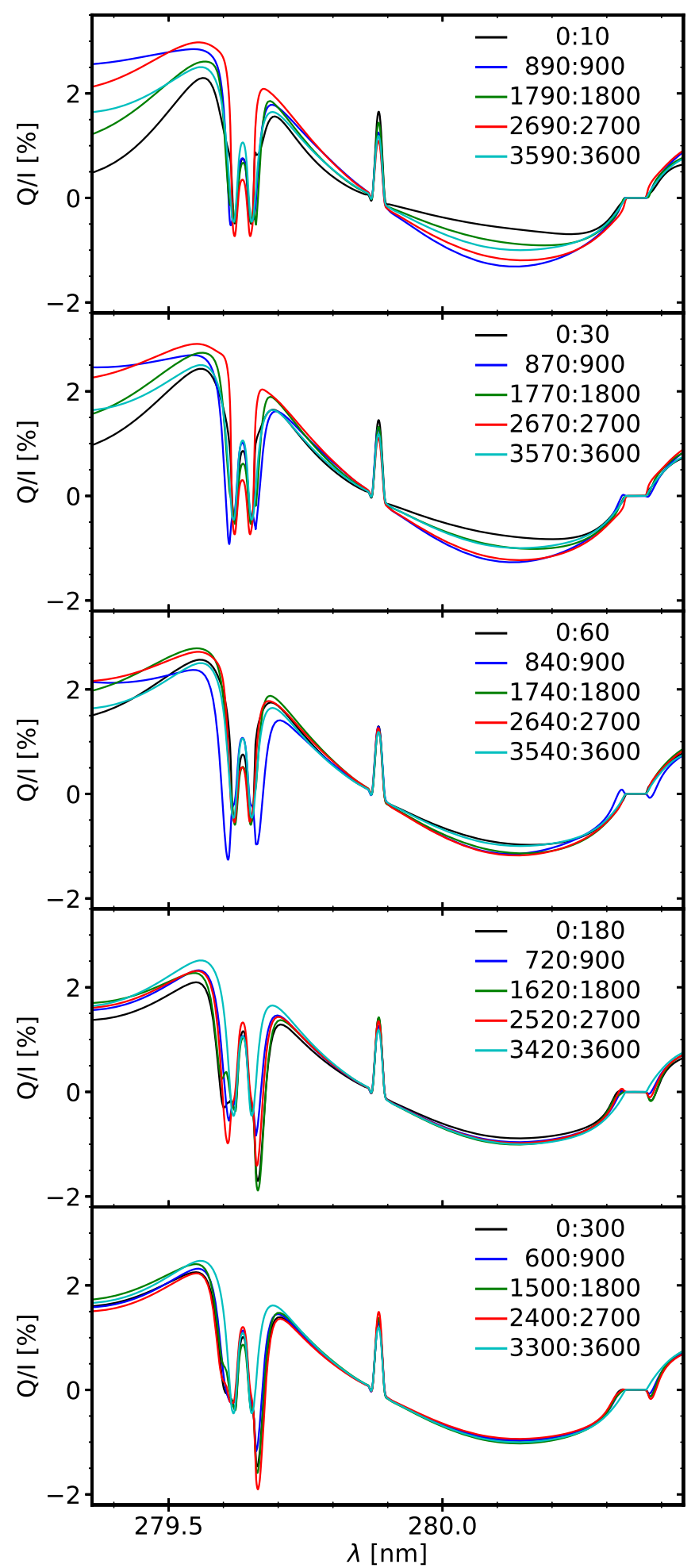

FIG. 18. - Time integral of the fractional linear polarization $Q / I$ profiles for the Mg II h-k doublet and UV triplet in the CS model for a LOS with $\mu=0.1$. Colors represent the starting and ending times of the integration interval, indicated in the legend of the panels. In the different panels, the profiles shown are the results of an integration of increasingly larger times, from top to bottom: $10 \mathrm{~s}, 30 \mathrm{~s}, 60 \mathrm{~s}, 180 \mathrm{~s}$, and $300 \mathrm{~s}$. 
The magnetic sensitivity across the Mg II h-k lines is caused by different physical mechanisms. The Hanle effect operates at the center of the $Q / I$ and $U / I$ profiles of the k-line and the subordinate lines, whereas the M-O effects determine the magnetic sensitivity of those polarization signals in the far wings. In contrast, the Zeeman effect practically dominates the circular polarization of all the lines in this spectral region.

The Stokes $V$ profiles of the h-k doublet show two lobes in each wing. The inner lobe is formed in the high chromosphere, while the outer one is formed at lower chromospheric heights. Therefore, the relative amplitudes of the two lobes can be used as a diagnostic of the relative strengths of the magnetic fields in the corresponding layers of the solar atmosphere.

As expected, the dynamics of the time-series model atmosphere considered in this work introduces some variability in the shapes and amplitudes of the Stokes profiles, as well as an enhancement of the radiation anisotropy. Nevertheless, when we take into account the typical exposure times of spectropolarimetric observations, this variability tends to be drastically reduced. In particular, for exposure times over 1 minute, the $Q / I$ polarization profile between the $\mathrm{k}$ and $\mathrm{h}$ transitions does not change appreciably along the time series.

The polarization signals and the physical effects described in this paper are within the observable range of instruments like CLASP-2. The modeling undertaken for this work is very complex, but it misses the additional symmetry breaking effects of 3D radiative transfer. Nonetheless, we are confident that the results presented in this work, and the effects discussed in this and previous papers, can already be verified or falsified at this stage through observations, without the need to further increase the realism and complexity of the model.

Finally, we want to emphasize the importance of designing and deplying space telescopes equipped with UV spectropolarimeters in the near future, in order to routinely attain the observational data necessary for the detailed study of the magnetism of the upper solar chromosphere and transition region. The complement of UV spectropolarimetric observations with the visible and IR observations already attainable from the ground will enormously increase the opportunities for new discoveries in both solar and stellar physics.

We acknowledge the funding received from the European Research Council (ERC) under the European Union's Horizon 2020 research and innovation programme (ERC Advanced Grant agreement No 742265).

\section{REFERENCES}

Allen, C. W. 1963, Astrophysical quantities

Alsina Ballester, E., Belluzzi, L., \& Trujillo Bueno, J. 2016, ApJ, 831, L15

-. 2018, ApJ, 854, 150

Auer, L. H., Rees, D. E., \& Stenflo, J. O. 1980, A\&A, 88, 302

Belluzzi, L., Landi Degl'Innocenti, E., \& Trujillo Bueno, J. 2013, A\&A, 551, A84

Belluzzi, L., \& Trujillo Bueno, J. 2012, ApJ, 750, L11

-. 2014, A\&A, 564, A16

Belluzzi, L., Trujillo Bueno, J., \& Štěpán, J. 2012, ApJ, 755, L2

Bohlin, J. D., Frost, K. J., Burr, P. T., Guha, A. K., \& Withbroe, G. L. 1980, Sol. Phys., 65, 5

Calvert, J., Griner, D., Montenegro, J., et al. 1979, Optical Engineering, 18, 287

Carlin, E. S., Asensio Ramos, A., \& Trujillo Bueno, J. 2013, ApJ, 764,40

Carlin, E. S., Manso Sainz, R., Asensio Ramos, A., \& Trujillo Bueno, J. 2012, ApJ, 751, 5

Carlsson, M., \& Stein, R. F. 1997, ApJ, 481, 500

Casini, R., del Pino Alemán, T., \& Manso Sainz, R. 2017a, ApJ, 835,114

-. 2017b, ApJ, 848, 99

Casini, R., \& Manso Sainz, R. 2016a, ApJ, 824, 135

-. 2016b, ApJ, 833, 197

Cunto, W., Mendoza, C., Ochsenbein, F., \& Zeippen, C. J. 1993, A\&A, 275, L5

De Pontieu, B., Title, A., \& Carlsson, M. 2014, Science, 346, 315

del Pino Alemán, T., Casini, R., \& Manso Sainz, R. 2016, ApJ, 830, L24

del Pino Alemán, T., Trujillo Bueno, J., Štěpán, J., \& Shchukina, N. 2018, ApJ, 863, 164

Fontenla, J. M., Avrett, E. H., \& Loeser, R. 1993, ApJ, 406, 319

Henze, W., \& Stenflo, J. O. 1987, Sol. Phys., 111, 243

Kano, R., Bando, T., Narukage, N., et al. 2012, in Society of Photo-Optical Instrumentation Engineers (SPIE) Conference Series, Vol. 8443, Proc. SPIE, 84434F

Kano, R., Trujillo Bueno, J., Winebarger, A., et al. 2017, ApJ, 839, L10

Kobayashi, K., Kano, R., Trujillo-Bueno, J., et al. 2012, in Astronomical Society of the Pacific Conference Series, Vol. 456, Fifth Hinode Science Meeting, ed. L. Golub, I. De Moortel, \& T. Shimizu, 233
Kramida, A., Yu. Ralchenko, Reader, J., \& and NIST ASD Team. 2018, NIST Atomic Spectra Database (ver. 5.5.6), [Online]. Available: http://physics.nist.gov/asd [May 2018]. National Institute of Standards and Technology, Gaithersburg, MD.

Landi Degl'Innocenti, E., \& Landi Degl'Innocenti, M. 1977, A\&A, 56,111

Landi Degl'Innocenti, E., \& Landolfi, M. 2004, Polarization in Spectral Lines (Kluwer Academic Publishers)

Manso Sainz, R., del Pino Alemán, T., Casini, R., \& McIntosh, S. 2019, ApJ, 883, L30

Manso Sainz, R., Roncero, O., Sanz-Sanz, C., et al. 2014, ApJ, 788,118

Mihalas, D. 1970, Stellar Atmospheres (W.H. Freeman and Company)

—. 1978, Stellar atmospheres /2nd edition/

Narukage, N., McKenzie, D. E., Ishikawa, R., et al. 2016, in Society of Photo-Optical Instrumentation Engineers (SPIE) Conference Series, Vol. 9905, Proc. SPIE, 990508

Pereira, T. M. D., Carlsson, M., De Pontieu, B., \& Hansteen, V. 2015, ApJ, 806, 14

Pereira, T. M. D., De Pontieu, B., Carlsson, M., et al. 2014, ApJ, 792, L15

Priest, E. 2014, Magnetohydrodynamics of the Sun

Rybicki, G. B., \& Hummer, D. G. 1991, A\&A, 245, 171

Sampoorna, M., \& Nagendra, K. N. 2015, ApJ, 812, 28

Sampoorna, M., Nagendra, K. N., \& Stenflo, J. O. 2017, ApJ, 844,97

Sigut, T. A. A., \& Pradhan, A. K. 1995, Journal of Physics B Atomic Molecular Physics, 28, 4879

Stenflo, J. O. 1980, A\&A, 84, 68

Strong, K. T., Saba, J. L. R., Haisch, B. M., \& Schmelz, J. T. 1999, The many faces of the sun : a summary of the results from NASA's Solar Maximum Mission (Berlin: Springer)

Trujillo Bueno, J., Landi Degl'Innocenti, E., \& Belluzzi, L. 2017, Space Sci. Rev., 210, 183

Trujillo Bueno, J., \& Manso Sainz, R. 1999, ApJ, 516, 436

Trujillo Bueno, J., Štěpán, J., \& Casini, R. 2011, ApJ, 738, L11

Trujillo Bueno, J., Štěpán, J., Belluzzi, L., et al. 2018, ApJ, 866, L15

Štěpán, J., \& Trujillo Bueno, J. 2016, ApJ, 826, L10

Woodgate, B. E., Tandberg-Hanssen, E. A., Bruner, E. C., et al. 1980, Sol. Phys., 65, 73 